\title{
Studien über den Einfluss des Dotters auf die Gastrulation und die Bildung der primären Keimblätter der Wirbelthiere.
}

\section{Selachier.}

Von

\author{
Dr. Paul Samassa,
}

Privatdocent in Heidelberg.

Mit Tafel XII-XIY.

Eingegangen am 27. Februar 1895.

Es bedarf wohl keiner besonderen Rechtfertigung, wenn ich im Nachfolgenden einige Beiträge zu dem im Titel angedenteten Problem zu liefern versuche. Nachdem in den meisten Arbeiten den palingenetischen Vorgängen, bezw. dem was man dafür hält, die Hauptaufmerksamkeit geschenkt wird, kann es nicht überflüssig sein, wenn einmal die Wirkung eines bestimmten cenogenetischen Faktors im Zusammenhange untersucht wird; indirekt werden die Resultate natürlich auch wieder der Phylogenie zu Gute kommen. Dass ich mich darauf beschränken muss, den Einfluss des Dotters auf bestimmte Vorgänge der ersten Entwickelung und auch dies nur innerhalb eines Typus zu untersuchen, gebietet der Umfang des Themas. Auch die Wahl der Thiergruppe wird wohl Niemand Wunder nehmen; denn in keiner Gruppe liegen die Verhältnisse für unsere Frage so günstig wie bei den Wirbelthieren. Einerseits finden wir alle Übergänge von dotterarmen Eiern bis zk den dotterreichsten, die im Thierreiche überhaupt vorkommen, vertreten; andererseits haben wir allen Grund zu der Annahme, dass in mehreren Zweigen des Wirbelthierstammes die gróße Dottermenge 
unabhängig erworben wurde; schließlich finden wir bei den Säugethieren zweifellos sekundär dotterarme Eier vor ${ }^{1}$. Ein nicht zu unterschätzender Vortheil ist es ferner, dass die Entwickelung rder Wirbelthiere bereits sehr genan durchforscht ist, wodurch die Behandlung einer bestimmten Einzelfrage sehr erleichtert wird.

Ein Übelstand liegt in einer anderen Richtung; man hat sich wobl kaum in einer anderen Thiergruppe so um die Frage interessirt, ob ein Gastrulastadium und Gastrulation in der Entwickelung vorkommt, wie bei Wirbelthieren; das Resultat entspricht aber diesen Bemühungen nur in so fern, als von den meisten Forschern das Vorkommen von Gastrulation auch bei den meroblastischen Eiern angenommen wird; was man aber dafür zu halten habe, darïber gehen die Meinungen weit aus einander. Es würde mich zu weit führen, wenn ich dies durch Litteraturnachweise eingehend belegen wollte; diese Thatsache ist ja sehr bekannt und zudem komme ich im Verlaufe meiner Einzeluntersuchungen darauf, sie in jedem Falle zu erweisen. Wenn ich nun auch nicht unterschätzen will, dass sich insbesondere bei den meroblastischen Eiern sowie bei Säugethieren mit ihren in vielen Dingen ähnlichen Verhältnissen sowobl der Beobachtung als auch der Deutung manche Schwierigkeiten in den Weg stellen, so glaube ich doch, dass ein Hauptgrund für die vielen Widersprüche darin liegt, dass der Gastrulationsbegriff den scharf begrenzten Inhalt verloren hat, den er unsprünglich besaß. Ich muss daher anf diesen Punkt etwas näher eingehen.

Als HAECKEL zuerst ${ }^{2}$ ) den Namen Gastrula * einfuihrte, belegte er mit diesem Namen ein Entwickelungsstadium, in dem er nach dem biogenetischen Gesetz den ontogenetischen Ausdruck einer Form sah, die im Laufe der Stammesgeschichte aufgetreten war und den Übergang zwischen den Protozoen und Metazoen bildete; er gab dieser Form den Namen *Gastraea «3). In einer späteren Arbeit finden wir folgende Definition der Gastrula:

»Ein einachsiger, hohler Körper ohne Anhänge, dessen einfache Höhle (Urdarm) sich an einem Pole der Achse durch eine Mündung.

1) Der Annahme Rabl's (Theorie des Mesoderms, Morphol. Jahrb. 15. Bd. 1889) von einem zweimaligen Dotterverlust in der zu den Säugethieren führenden Reihe kann ich mich nicht anschließen.

2) HAECKEL, E., Die Kalkschwämme. 1872.

3) Hazckel, E., Die Gastraeatheorie, die phylogenetische Klassifikation des Thierreiches und die Homologie der Keimblätter. Jenaische Zeitschr. f. Naturw. Bd. VIII. 1874. 
öffnet (Urmund), und dessen Körperwand aus zwei Zellenschichten oder Blättern besteht: Entoderm oder Gastralblatt und Exoderm oder Dermalblatt. *

Wenn auch nicht in der Definition selbst, so nimmt doch sonst überall HAECKEL Bezug darauf, dass er in der Gastrulaform die ontogenetische Rekapitulation der phylogenetischen Gastraea erblickt. Mit Rücksicht auf den terminologischen Gebrauch, neu eingeführte Namen nur in der Bedeutung zu gebrauchen, die ihnen vom Autor derselben beigelegt wurde, wie auch aus ZweckmäBigkeitsgrüinden empfiehlt es sich, die Ausdrïcke »Gastrula« und »Gastrulation« nur zu gebrauchen, wenn man sich auf den Boden der Gastraeatheorie stellt; denn die Verwirrung ist unausbleiblich, wenn einige Forscher nur homologe Bildungen mit dem Ausdrucke Gastrula zusammenfassen, während andere ihrer Ansicht nach analoge Formen damit bezeichnen, da in jedem Falle der Kreis der darunter subsumirten Einzelfälle einen anderen Umfang haben wird.

Nachdem wir uns nun dahin geeinigt haben, nur das als Gastrulastadium zu bezeichnen, was in der Ontogenie als Rekapitulation des phylogenetischen Gastraeastadiums angesehen werden kann, frag't es sich, wie wir dieses ontogenetische Stadium erkennen können? Ist dasselbe so deutlich ausgesprochen, dass es ganz der gegebenen Definition entspricht, dann kann dies natirlich keine Schwierigkeiten haben; ist dasselbe aber durch cenogenetische Einfluisse verändert, dann können uns vielleicht die Bildungsvorgänge, die zum Gastrulastadium führen, einige Anhaltspunkte geben. Denn ebenso, wie die Gastrula nur der Ausdruck ontogenetischer Bildungsprocesse ist, ist die Gastraea der Ausdruck einer phylogenetischen Reihe von Veränderungen; und es fragt sich, welche Reihe wir als zur Gastraea führend annehmen, wenn wir den palingenetischen Modus der Gastrulabildung feststellen wollen. Diese Frage ist um so wichtiger, als sie im engsten Zusammenhange steht mit der Bildung der primären Keimblätter, die wir ja in der Gastrula bereits fertig vorfinden.

Es giebt drei Möglichkeiten, wie wir uns die Bildung der Gastraea vorstellen können; die eine wird von HAEckEL vertreten I); demnach soll die "Gastraea« aus der »Blastaea hervorgegangen sein, einer kugligen, allseitig mit Zellen bedeckten Form, bei der jedoch bereits eine Scheidung von lokomotorischen und nutritiven Zellen

1) Hanckes, E., Die Gastrula and die Eifurchung der Thiere. Jenaische Zeitschr. f. Naturgesch. IX. Bd. 1875. 
stattgefunden hat. Da die letzteren an einem Pole angesammelt sind, so läge es im Interesse ihrer Funktion, wenn sie nicht die Oberfläche bedecken, sondem einen Hohlraum auskleiden, und diesem Vortheil verdanke die Einstülpung ihre Entstehung. Diese Annahme hat offenbar zwei Schwächen: erstens ist nicht einzmsehen, wie sich an einer sich in allen Richtungen bewegenden Kugel die Ernährungszellen gerade an einem Pol ansammeln können, da eine Vertheilung derselben iiber die ganze Kugeloberfläche doch viel zweckmäßiger wäre; zweitens können die ersten Stadien der Einstülpung, die doch ganz allmählich im Laufe der Phylogenie aufgetreten sein müssen, unmöglich den Vortheil gehabt haben, von dem HaEckel spricht; beide Einwände werden bereits von Ray LaNkester ${ }^{1}$ ) and BÜTschLI ${ }^{2}$ ) gegen die HaEckeL'sche Auffassung geltend gemacht.

Die zweite Hypothese über die Vorstufen der Gastraea rührt von Bürschur ${ }^{2}$ ) her; er nimmt statt der Blastaea eine Form von Plattengestalt, die "Plakaea an, bei der die beiden primären Keimblätter bereits als zwei Zellschichten gesondert waren; indem diese Platte sich einkrümmt, kommen wir zur Gastraeaform. Die Annahme BüTsCHLI's hat jedenfalls für sich, dass sie in der Ontogenie fast aller Thiergruppen starke Stützen findet; ihre Schwierigkeit, die Bürschur selbst zugiebt, liegt aber darin, dass die Entstehung des Entoderms schwer zu erklären ist.

Eine dritte Annahme wurde, so viel ich sehe ${ }^{3}$ ), in dieser Form wenigstens noch nicht aufgestellt. Cuaus hat in seiner gegen die Gastracatheoric gerichteten Schrift') die Bildung der Gastrula darauf zurückzufïhren gesucht, dass beim Wachsthum der Blastula das Verhältnis zwischen Oberfäche und Volumen immer ungünstiger wird ${ }^{5}$ und daher eine Einstillpung erfolgt, die die Gastrula erzengt.

1) RaY Lankester, Notes on embryology and classification. 1877.

2) Bütschli, 0., Bemerkungen zur Gastraeatheorie. Morphol. Jahrb IX. Bd. 1884.

3) Ich muss diese Einschränkung allgemein für diese theoretische Diskussion machen, denn tam Alles, was in dieser lirage gesagt worden ist, zu kennen, miisste man die gesammte Litteratur der letzten 20 Jahre daranfhin durchsuchen, da ja derartige Erörterungen meist in Specialarbeiten verstrent siad.

4) C. Claus, Die Typenlehre uad E. Hagcker's sog. Gastrasatheorie. Wien 1874.

5) Da die Volumina wachsen wie die dritten, die Oberfächen wie die zweiten Potenzen der Radien. Anf die Bedeutung dieses Verhältnisses für die Formengestaltung im Thierreiche wurde zuerst von LEUCKART aufmerksam gemacht: R. Leuckart, Über einige Verschiedenheiten der Thiere nud Planzen. 
Dieses Princip hat in seiner Anwendung anf die Ontogenie den Übelstand, den jeder mechanische Erklärungsversuch ontogenetischer Vorgänge hat; denn da dieses Princip auch bei dotterlosen Formen nicht immer in Wirksamkeit tritt, so ist sein Einfluss offenbar an gewisse Bedingungen gekniipft, die nur durch Vererbung zu erklären sind. Hingegen scheint mir, dass wir sehr gut die phylogenetische Entstehung der Gastraea dadurch erklären können; wir müssen dabei von einer Blastaea ausgehen, deren Zellen alle gleich waren, und deren Inneres von einer homogenen Gallerte erfillt war. Nehmen wir nun an, dass dieser Organismus die Tendenz hatte immer weiter zu wachsen, so musste ein Punkt kommen, wo das immer ungünstiger werdende Verhältnis zwischen Oberfläche und Volum diesem Bestreben ein Ziel setzte. Ein weiteres Wachsthum war dann nur möglich unter allmählicher Verdrängung der Gallerte, also durch Einstulpung eines Theils der Blastaeaoberfläche; dabei ist klar, dass jeder kleinste Schnitt in dieser Richtung das Verhältnis zwischen Oberfläche und Volumen verbessern und daher dem Organismus von Vortheil sein musste. Eine Arbeitstheilung zwischen den Zellen würde aber erst erfolgen, nachdem die Einstülpung ganz vollzogen ist, indem die die Höhle auskleidenden Zellen nutritive, die der Außenwelt zugekehrten lokomotorische Funktionen übernehmen. Die Bildung der beiden primären Keimblätter musste also in diesem Falle als eine Folge der Gastraeabildung angesehen werden, während sie nach den Annahmen HAEckEL's und BürschLI's derselben bereits vorausgegangen ist.

Dadurch, dass wir drei verschiedene Entwickelungsreihen gefunden haben, die zur Gastraea geführt haben können, wird natürlich die Aufgabe, im einzelgeschichtlichen Fall festzustellen, was als Gastrulation anzusehen ist, nicht erleichtert; je nach dem Standpunkt, auf den man sich stellt, wird auch die Beurtheilung, ob ein Vorgang oder ein Stadium palingenetisch ist oder nicht, verschieden ausfallen. Um dies an einem Beispiel zu zeigen, wird bei Amphioxus das achtzellige Stadium nach BüTschLr als palingenetisch angesehen werden mïissen, das Blastulastadium als cenogenetisch; hingegen ist nach HAECKEL die so frïh erfolgende Differenzirung:

Arch. f. Naturgesch. 1851. S. dariber ferner: BERGMANN und Leuckan', Vergl. Anatomie und Physiologie. 1852. J. A. Rrosk, The Correlation of the Volumes and Surfaces of Organisms. Contrib. of the zool. Labor. Úniv. of Pennsylv. VI. 1893. Für den Hinweis auf die beiden letzteren Werke bin ich Herrn Prof. Bürschu zu Dank verpflichtet.) 
der Keimblätter cenogenetisch, die Blastula völlig palingenetisch, hingegen nach meiner Auffassung ist auch die Blastula noch cenogenetisch in so fern, als an ihr die Keimblätter schon geschieden sind. Andererseits wird es aber auch Fälle geben, wo wir unter jeder Voraussetzung einen bestimmten Vorgang als cenogenetisch ansehen müssen, wenn wir überhaupt auf dem Boden der Gastraeatheorie stehen, z. B. die Bildung des Entoderms durch Immigration u. A. Da wir aber nun doch unter allen Umständen eine Reihe von Voraussetzungen annehmen müssen, wenn wir von der Ontogenie auf die Phylogenie schließen, so scheint es mir das kleinere Übel zu sein, wenn wir diesen Voraussetzungen eine weitere hinzufügen, als wenn wir mit Begriffen operiren, denen jeder scharfbegrenzte Inhalt fehlt. Und dies scheint mir in der That mit dem Worte "Gastrulation" der Fall zu sein, wenn man es so gebraucht, wie es sehr häufig geschieht, indem man einen Vorgang in der Entwickelung eines Thieres damit bezeichnet, der eine gewisse $\ddot{A} h n-$ lichkeit mit dem Vorgange hat, der bei einem verwandten Thiere eben so genannt wird; wobei gewöhnlich ganz außer Acht gelassen wird, dass es sich doch um etwas handelt, das bei allen Metazoen homolog ist und daher auf einen bestimmten phylogenetischen $\mathrm{Zu}-$ stand bezogen werden muss. Zudem fällt es ja doch gar nicht schwer, die Beschreibung eines Vorganges von seiner im Sinne der Gastraeatheorie gegebenen Deutung desselben zu unterscheiden und dadurch die Thatsachen auch dem annehmbar zu machen, der mit der Deutung nicht einverstanden ist.

Dasselbe, was im Vorstehenden ïber die Gastrulation und das Gastrulastadium gesagt ist, gilt meines Erachtens auch für die damit eng verknüpften Begriffe »Urmund « und »Urdarm «; man sollte nur solche Bildungen damit belegen, die man den gleichbenannten Bildungen der Gastraea für entsprechend hält. Nun wird aber gerade das Wort Urmund sehr häufig so angewendet, dass man eine, oft gar nicht wesentliche Eigenschaft desselben als genügend erachtet, um eîner Bildung diese Bezeichnung beizulegen. So ist es zwar sicher, dass wir annehmen müssen, dass bei der Gastraea am Urmund die Grenze der beiden primären Keimblätter liegt; das ist aber jedenfalls nicht das wesentlichste Kennzeichen desselben, was schon daraus hervorgeht, dass sowohl nach der Annahme HaEckel's als auch nach der BüTscrur's schon vor dem Gastraeastadium eine derartige Grenze bestanden haben muss. Es hat daher keinen Sinn, eine derartige Grenze der beiden primären Keimblätter als Urmund zu 
bezeichnen, wie es z. B. Ү. DavmoofF ${ }^{1}$ ) bei Distaplia thut, wenn derselben die Haupteigenschaft des Urmunds als Öffnung des Urdarms nach außen fehlt. Die Richtigkeit meiner Auffassung ergiebt sich auch daraus, dass z. B. bei Amphibien die Grenze der primären Keimblätter (Umwachsungsrand des Ektoderms) mit der Öffnung des Urdarms nach außen (Urmund) gar nicht zusammenfällt. Ich verstehe daher nicht, wie 0 . HerTwig ${ }^{2}$ ) in derselben Arbeit, in der er sich dagegen verwahrt, dass der Umwachsungsrand der Keimscheibe bei Amnioten als Urmund aufzufassen sei, dem Umwachsungsrand des Ektoderms bei Amphibien, der principiell eine ganz ähnliche Bildung ist, diese Deutung giebt. Diese Arbeit beweist auch, dass es durchaus nicht gleichgiultig ist, wie man derartige Ausdrücke anwendet; denn von der Homologie des Urmundes bei allen Metazoen ausgehend, zieht HerTwig zwischen der Anordnung des Nervensystems bei Wirbelthieren und Coelenteraten Vergleiche, die bei richtiger Anwendung des Urmundbegriffs ohne Weiteres hinfällig werden.

Die erwähnte Arbeit $O$. HerTwIG's veranlasst mich noch zu einer weiteren Bemerkung; wenn, wie wir annehmen, die Gastrulation die ontogenetische Rekapitulation der zur Gastraea führenden Entwickelungsreihe darstellt, dann. muss dieser Vorgang bei allen Abkömmlingen der Gastraea wesentlich derselbe sein, so weit er eben palingenetisch ist. Nun ist es gewiss möglich, dass innerhalb eines Typus der Urmund ganz bestimmte Beziehungen zu den Anlagen anderer, phylogenetisch jüngerer Organe hat; dies lässt sich aber nur empirisch feststellen, indem man die ganze Gruppe daraufhin untersucht. Hertwig jedoch stellt die Eigenschaften des Urmunds für zwei Klassen der Wirbelthiere (Amphioxus und Amphibien) fest, zieht überdies in die Definition ganz unbewiesene Hypothesen (wie die Concrescenztheorie) hinein und fordert nun, dass der Urmund bei allen Wirbelthieren diese Eigenschaften haben müsse. Es kann aber doch, wie ich glaube, keinem Zweifel unterliegen, dass der Ort der Anlage phylogenetisch jüngerer Organe Verschiebungen erfahren kann, die vom Urmund ganz unabhängig sind, und dass die Gastrulation cenogenetische Beeinflussungen erfährt, die mit ersteren nichts zu thun haben.

Es erübrigt mir noch, etwas über die Bezeichnung »Blastoporus *

1) DavidofF, M. v., Untersuchungen zur Entwickelungsgeschichte der Distaplia magnilarva. Mitth. d. Zool. Stat. Neapel. IX. Bd.

2) Hertwig, O., Urmund und Spina bifida. Archiv f. mikr. Anat. XXXIX. Bd. 1892. 
zu sagen; dieselbe wurde von RAY LANKESTER ${ }^{1}$ ) in einem Sinne aufgestellt, die von der Bedeutung des HaEckeL'schen Urmunds durchaus abweicht; insbesondere giebt LANKESTER die Homologie des Urmunds innerhalb der Metazoen nicht zu und sieht in demselben eine gelegentlich auftretende cenogenetische Bildung. Toh halte es daher nicht für angebracht, den Ausdruck Blastoporus in demselben Sinne wie Urmund zu gebrauchen, was vielfach geschieht. Man sollte vielmehr diesen Ansdruck zum Gebrauch jenen Forschern reserviren, welche die Gastraeatheorie nicht anerkennen und den Urmund ähnlich auffassen wie LaNkEsteR.

Schließlich dürfte eine Bemerkung nicht überflüssig sein: HAECKEL ${ }^{2}$ ) suchte die Richtigkeit der Gastraeatheorie durch eine möglichst extensive Beweisführung zu erhärten; dabei beging er in seiner vorgefassten Meinung, ibberall eine Gastrulation finden zu müssen, sowohl in seinen eigenen Beobachtungen als auch bei der Deutung. der Beobachtungen Anderer manchen Irrthum. Für mich liegt aber die Stärke der Gastraeatbeorie vor Allem darin, dass sie von allen Hypothesen über den Ursprung der Metazoen meines Erachtens die größte innere Wahrscheinlichkeit hat; und irgend eine Vorstellung über den Ursprung der Metazoen mïssen wir uns schließlich doch machen, mag dieselbe im Übrigen auch noch so hypothetisch sein. Wenn die Gastraeatheorie außerdem in der Ontogenie der meisten ursprünglichen und dotterlosen Formen eine Bestätigung findet, so kann uns das völlig genügen. Wie sich die Gastrulation unter dem Einflusse cenogenetischer Veränderungen, wie insbesondere in Folge des Anwachsens des Nahrungsdotters gestaltet, ist eben zu untersuchen; sollte sich dabei auch herausstellen, dass die Gastrulation in manchen Fällen ganz wegfällt, so kann das nach meiner Meinung gegen die Gastraeatheorie gar nichts beweisen. Diese Erwägungen werden mich davor bewahren, eine Gastrulation um jeden Preis zu finden, ein Bestreben, das gerade in der Embryologie der Wirbelthiere eine große Rolle spielt.

Weun ich im Vorstehenden der Gastrulation und dem was damit zusammenhängt eine so eingehende Würdigung zu Theil werden ließ, so geschah dies hauptsächlich desshalb, weil wir in der Litteratur, die die erste Entwickelung von Wirbelthieren behandelt, ständig diese Bezeichnung und Deutung antreffen; es scheint da-

1) 1. c.

2) Hafcket, E., Die Gastrula und die Eifurchung der Thiere. Jen. Zeitschr. f. Naturgesch. IX. Bd. 1875. 
her unerlässlich, einen Maßstab zu gewinnen, nach dem man diese Deutungen misst. Hingegen liegt es mir durchaus fern, bei der mir gesteckten Aufgabe von der Gastraeatheorie als Voraussetzung auszugehen; so sehr ich auch den heuristischen Werth derselben anerkenne, so wenig verkenne ich deren hypothetischen Charakter. Zudem ist es, wenn wir z. B. die Entwickelung eines Selachiers mit der des Amphioxus vergleichen, ganz gleichgültig, ob wir letztere als ursprünglich im Sinne der Gastraeatheorie deuten oder nicht; im Verhältnis zu der des Selachiers ist sie es jedenfalls and die Differenzen, die wir vorfinden, können wir auf Rechnung des Dotters setzen, sofern wir eben andere Ursachen fur dieselben auszusehließen in der Lage sind. Ich werde auch immer diese Differenzen unabhängig von der durch die Gastraeatheorie gebotenen Deutung zusammenfassen und dadurch meine Resultate für Jedermann verwerthbar machen, er stehe im Übrigen zur Gastraeatheorie wie er wolle.

Es scheint mir hier noch am Platze, auf eine Arbeit Lwofw's ${ }^{1}$ ) einzugehen, in der sich theoretische Auseinandersetzungen finden, die in mancher Beziehung von ähnlichen Gesichtspunkten ausgehen, wie die, die ich im Vorstehenden entwickelt habe; die Resultate, zu denen Lwowf kommt, sind freilich von meinen sehr verschieden. Tch stimme LwoFF vollständig bei, wenn er die Unzuträglichkeiten hervorhebt, zu denen der Gebrauch des Wortes Gastrulation seitens der meisten Forseher gefuhrt hat; wenn man z. B. bei Amnioten so weit gekommen ist, dass die Gastrulation mit der Bildung des Darms gar nichts zu thun habe und blok die Bildung von Chorda und Mesoderm bezweckt; was, wenn man nur konsequent sein wollte, zu der Schlussfolgerung führen müsste, dass der Darm der Amnioten gegenüber dem der niederen Wirbelthiere eine Neubildung ist. Den Anschaungen aber, die Lwofr an Stelle der herrschenden setzen will, kann ich mich nicht anschließen. Nach Lwoff ist das Einzige, was man bei Wirbelthieren als Gastrulation ansehen könnte, die Umwachsung der Makromeren holoblastischer Eier durch die Mikromeren; eine Einstiilpung findet nur bei Amphioxus statt, wo durch dieselbe die Entodermzellen ins Innere verlagert werden. Den Begriff »Urdarm « and „Urmund « glaubt er entbehren zu müssen; der Urdarm zerfällt für ihn einfach in einen ekto- and entodermalen Antheil. Auf die Unterscheidung der beiden Keimblätter kommt

1) Lwofr, B., Die Bildung der primären Keimblätter und die Entstehung der Chorda und des Mesoderms bei den Wirbelthieren. Bulletin d. 1. soc. imp. d. nat. d. Moscon. A. 1894. Nr. 1 n. 2. 
also offenbar Alles an; sehen wir wie LwofF dabei zu Werke geht. Bezüglich des Entoderms sagt LwoFf (pag. 96): »Im Folgenden werde ich jene Zellen Entodermzellen nennen, aus welchen der Darm entsteht; « etwas vorher aber heißt es: »so muss man bei der Bestimmung der primären Keimblätter zunächst ins Klare bringen, welche Elemente oder welche Schicht den Darm bildet. Diese Schicht muss man Entoderm nennen, gleichviel ob von diesen Zellen noch etwas Anderes außer dem Darm gebildet wird oder nicht. * Wenn aber nur die Zellen Entodermzellen sind, aus denen der Darm entsteht, so ist nicht gut einzusehen, wie aus denselben noch etwas Anderes entstehen kann als der Darm. In der praktischen Anwendung dieser Theorie ergeben sich dann dieselben Widerspriiche; so heißt es z. B. von Petromyzon: "Da die inneren, von den Mikromeren umwachsenen Zellen den Darm bilden, so kann man sie mit vollem Recht als Entoderm bezeichnen." Später stellt sich aber heraus, dass auch ein Theil des Mesoderms aus dem Entoderm entstehen soll. Woran sind dann aber die Entodermzellen zu erkennen; vielleicht daran, dass sie ron den Mikromeren umwachsen sind? Dann sind also die Mikromeren wohl Ektoderm; von einem früheren Stadium wird dies aber ansdrücklich geleugnet; es heißt: »ich habe anch schon erwähnt, dass die Mikro- und Makromeren der frühen Stadien mit den Ektoderm- und Entodermzellen keineswegs identificirt werden können. Woher kommt dann auf einmal die Berechtigung, die Keimblätter nach der Größe ihrer Zellen zu unterscheiden? Ich vermisse bei Lwoff die Konsequenz; wenn er bloß die Zellen, die den Darm bilden, als Entoderm bezeichnen würde, den ganzen Rest als Ektoderm, so wäre das schließlich ein Standpunkt; freilich müsste man sich fragen, ob es dann der Keimblätter überhaupt bedarf und $o b$ es nicht einfacher wäre, die Anlage des Darmes der aller übrigen Organe einfach gegenüberzustellen. Wie man aber den Begriff des Urdarmes als überflüssig zurückweisen kann, um an dessen Stelle eine völlig unklare Unterscheidung der Keimblätter zu setzen, ist mir unverständlich. Mit Unrecht beruft sich hierbei LwoFF auf die Autorität Huxley's, um seine Auffassung zu stiitzen; denn gerade in der von Lwof citirten Stelle weist Huxlex auf die Übereinstimmung zwischen dem ausgebildeten Hydrozoon und dem $\gg$ sackförmigen * Embryonalstadium höherer Thiere hin. Das ist aber vollständig der Standpunkt der Gastraeatheorie, nur dass dieselbe an die Stelle des Hydrozoons einen noch einfacher gebauten Organismus setat. Dass aber aus der inneren 
Schicht des »sackförmigen« Embryos bloB der Darm entstehen dürfte, ist nicht gesagt, das ist vielmehr in jedem Falle erst festzustellen.

Falls die Beobachtungen Lworf's über die Entwickelung: der Wirbelthiere mit totaler Furchung richtig sind, so folgt meiner Ansicht nach aus denselben weiter nichts, als dass die Auffassung der Makromeren als Entoderm und der Mikromeren als Ektoderm (bezw. bei Amphioxus der vegetativen und animalen Furchungszellen), welche von den meisten Forschern vertreten wird, irrthümlich ist, dass vielmebr beide Zellarten zur Bildung des Urdarms verwendet werden, wobei die Mikromeren die Chorda und einen Theil des Mesoderms bilden. Für Selachier werde ich übrigens in dieser Studie nachweisen können, dass die Beobachtungen, die Lwofw bei denselben im Sinne seiner Theorie gemacht zu haben meint, durchaus irrthümlich sind, und für die Teleostier ist es mir nach meinen bisherigen Untersuchungen bereits sehr wahrscheinlich. Nach diesen Erfahrungen wird man es dann wohl begreifen, dass ich auch die übrigen »Entdeckungen« LworF's etwas skeptisch aufnehme.

\section{Die Dotterkerne.}

Ein wesentlicher Theil meiner Aufgabe ist es, die Stellung zu ermitteln, welche die Dotterkerne (Merocyten, RÜCKERT) den Keimblättern gegenüber einnehmen. Ich habe schon an anderer Stelle ${ }^{1}$ ) ausgeführt, dass ich mich jener weit verbreiteten Auffassung, welche in den Zellen, deren Aufgabe die Assimilirung des Dotters ist, unter allen Umständen Entoderm sieht, nicht anschließen kann; HAECKEL, giebt dieser Ansicht einen prägnanten Ausdruck, indem er sagt ${ }^{2}$ ): „Da wir vom phylogenetischen Gesichtspunkte aus den gesammten Dotter überhaupt als Entodermprodukt auffassen, so müssen wir auch jene Dotterzellen als Zellen des Entoderms oder des Darmblattes im weiteren Sinne betrachten. N Nun ist dagegen vor Allem zu bemerken, dass der Dotter iberhaupt nicht das Produkt eines Keimblattes, also auch nicht des Entoderms ist, sondern ein Produkt der Eizelle und in zweiter Linie des mütterlichen Körpers. Einem Keimblatte ist der Dotter erst dann zuzurechnen, wenn er in ein solches aufgenommen worden ist; nun könnte man ja allerdings annehmen, dass beim ersten Auftreten des Dotters in der Stammes-

1) Samassa, P., Die Keimblätterbildung bei den Cladoceren. II. Archiv f. mikr. Anat. XLI. Bd. 1893.

2) Hacckes, E., Die Gastraea und die Eifurchung der Thiere. Jenaische Zeitschr. f. Naturgesch. IX. Bd. 1875. 
geschichte die Aufnahme desselben dem Entoderm zufiel; es ließe sich aber auch die Hypothese vertreten, dass der Dotter ursprünglich auf beide Keimblätter vertheilt war. Nehmen wir aber selbst das Erstere an, so folgt daraus in keiner Weise, dass sich dieser Zustand ïberall erhalten haben muss; denn wie beim ausgebildeten Organismus eine physiologische Funktion auf andere Organe oder auf eine Neubildung übergehen kann, so ist das ebenso bei der Funktion der Dotterresorption im Embryonalleben der Fall; sie kann auf ein anderes Keimblatt ibergehen, oder auch auf Elemente, die die Zurechnung zu einem Keimblatt gar nicht gestatten; gerade die Selachier bilden einen guten Beleg für diese Auffassung. Ich gebe zunächst eine Übersicht über die Angaben, welche bisher uiber Herkunft und Schicksal der Dotterkerne gemacht wurden. BALFOUR $^{1}$ ) war offenbar über die erste Entstehung derselben im Zweifel; er giebt zwar in seiner Monographie an, dass dieselben frei im Dotter entstiunden, setzt jedoch in der vergleichenden Embryologie ein Fragezeichen zu dieser Angabe. Während der Furchung: sollen dieselben Zuwachs zu den Furchungszellen liefern, indem der Dotter sich um einzelne Dotterkerne herum abgrenzt, wodurch echte Furchungskerne entstehen. Nachdem der Hohlraum aufgetreten ist, den BALFoUn als Furchungshöhle bezeichnet und der ursprünglich direkt an den Dotter grenzt, erhält derselbe durch Abschnürung von Zellen um die Dotterkerne herum eine ventrale Wand; diese verdrängt dann allmählich die Furchungshöhle, ein Vorgang, der von BALFoun als Gastrulation aufgefasst wird. Schließlich liefern Dotterzellen auch noch die ventrale Wand des Darmrohrs und betheiligen sich an der Blutbildung.

SchuLrz ${ }^{2}$ ) nimmt an, dass die Dotterkerne vou den peripher gelegenen Furchungszellen abstammen. Der größte Theil der Furchungszellen geht in das Ektoderm über, einige wenige, welche übrig bleiben, bekommen reichlichen Zuwachs von Dotterzellen und bilden das Entoderm; aus Dotterzellen entsteht auch noch das Blut.

C. K. Hormana ${ }^{3}$ ) hingegen spricht den Dotterkernen jede Betheiligung am Aufbau des Embryonalkörpers ab; wenn diese Angaben

1) Balfour, F. M., A monograph on the derelopment of Elasmobranch Fishes. London 1878.

2) Schultz, A., Beitrag zur Entwickelungsgeschichte der Knorpelfische. Arch. f. mikr. Anat. XIII. Bd, 1877.

3) Hofmmans, C. K., Contributions à l'histoire du developpement des Plagiostomes. Arch. Neerland d. Seiene ex. et nat. T. XVI. 1881. 
bei anderen Autoren wenig Beachtung fanden, so liegt das wohl hauptsächlich daran, dass er die Übereinstimmung mit den Teleostiern sehr betonte, die Entdeckung, die er aber iiber die Herkunft der Dotterkerne bei denselben gemacht zu haben glaubte, sich sehr bald als irrthümlich herausstellte.

In seiner ersten Arbeit ${ }^{1}$ fasst Rëckent die Dotterkerne mit dem sie umgebenden Plasma als Zellen auf, denen er den Namen Merocyten giebt; sie sollen durch eine Theilung der Furchungszellen entstehen, die der Theilung in animale und vegetative Furchungskugeln holoblastischer Eier entspricht. Während der Furchung liefern sie noch einiges Material zu den Furchungszellen; ihre Mauptthätigkeit beginnt aber bei der Keimblattbildung. Während nach der Ansicht RüCKERT's die Furchungszellen völlig in der Bildung des Ektoblast aufgehen, liefern die' Merocyten dèn gesammten primären Entoblast, indem am Boden der Furchungshöhle Zellen aus ihnen entstehen, die sich im Bereich des Urdarmes zu einer epithelialen Schicht zusammenfiugen; im übrigen Bereich der Keimscheibe stellen sie die Keimlager dar für die Bildung des Mesenchyms und des Blutes. Außer dem Ektoderm, das ïbrigens nach Rückent auch Abkömmlinge von Merocyten enthält, entsteht also Alles aus den Merocyten.

SWAEN 2), der allerdings nur spätere Stadien untersucht hatte, nimmt gleichfalls eine Betheiligung der Dotterzellen an der Entodermbildung" an.

Einen großen Fortschritt bedeutet die Entdeckung Kastscrenko's ${ }^{3}$, dass bereits im Stadium von zwei Furchungszellen eine Anzahl von Dotterkernen vorhanden ist. Über die Herkunft derselben spricht dieser Autor in einer späteren Mittheilung ${ }^{4}$ ) folgende Meinung aus: „der erste sogenannte Furchungskern theilt sich wiederholt, in Folge dessen ein vielkerniges Plasmodium gebildet wird; dasselbe wird nachber allmählich vom Centrum nach der Peripherie aus in einzelne Zellen getheilt. * Des Weiteren liefern die Dotterkerne während der Furchung Furchungszellen; bezüglich der. Betheiligung derselben an der Blut- und GefäBbildung ist Kastschenko zweifelhaft. Da er übrigens das ganze

1) Rückert, J., Zur Keimblattbildung bei Selachiern. München 1885.

2) SWAEr, A., Études sur le developpement de la Torpille. Archires de Biologie. VII. Bd. 1887.

3) Kastschenzo, N., Zur Frage über die Herkunt der Dotterkerne im Selachierei. Anat. Anz. III. 1888.

4) Kastschenko, N., Znr Entwickelungsgeschichte des Selachierembryos. Anat. Anz. III. 1888. 
gastrale Entoderm durch Einstülpung des Blastoderms am Embryonalrande der Keimscheibe entstehen lässt, so ist offenbar im Vergleich zu der Darstellung RückERT's der Antheil der Dotterkerne am Aufbau des Embryos bereits einigermaßen eingeschränkt.

Bald darauf konnte RüCKERT ${ }^{1}$ ) die Angaben Kastschenko's in Bezug auf das erste Auftreten der Dotterkerne bestätigen; er machte aber auch noch einen Schritt weiter, indem er eine Keimscheibe beschrieb, in der neben einem einzigen echten Furchungskern (Holocytenkern, RüCKERT) bereits mehrere Dotterkerne vorhanden waren. Im Gegensatz zu der Auffassung Kastschenko's konnte er nachweisen, dass bis zum 128 zelligen Stadium bei Torpedo weder Furchungszellen aus Merocyten entstehen, noch auch umgekehrt. Bezüglich der Abstammung der Dotterkerne nimmt er nunmehr an, dass der durch Vereinigung ron männlichem und weiblichem Vorkern entstehende Furchungskern durch Theilung je einen Stammkern für die Merocyten und für die Furchungszellen (Holocyten) liefert. Im Übrigen hält er aber an seiner früheren Auffassung über die Betheiligung der Merocyten am Aufbau des Embryonalkörpers fest.

Eine spätere Arbeit RückerT's ${ }^{2}$ ) bringt die entscheidende Wendung in der Beurtheilung der Merocyten, indem RückeR'T nachweist, dass schon vor der Vereinigung der Vorkerne eine Anzahl von Kernen in der Keimscheibe vorhanden ist; mit großer Wahrscheinlichkeit konnte RückERT nachweisen, dass diese Kerne von überschüssigen Spermatozoen, die in das Ei eingedrungen sind, herstammen. $\mathrm{Ob}$ aber alle Merocyten und insbesondere die späterer Stadien $\mathrm{Ab}$ kömmlinge von Samenfäden sind, lässt er hier noch unentschieden.

In einer ein Jahr später erschienenen Mittheilung ${ }^{3}$ ) diskutirt Rückert die Möglichkeit, dass die Merocyten von Zellen des mütterlichen Körpers abstammen, hält dies zwar für die am periphersten gelegenen für nicht ganz ausgeschlossen, aber doch für sehr unwahrscheinlich. Hingegen bringt er einen neuen Beweis bei für die Entstehung derselben aus Samenfäden durch die Beobachtung, dass die Dotterkerne bei Torpedo nur halb so viel Chromosomen besitzen als die Furchungskerne. RÜckERT zieht nunmehr auch

1) Rückert, J., Weitere Beiträge zur Keimblattbildung bei Selachiern. Anat. Anz. IV. 1889.

2) Rückert, J., Zur Befruchtung des Selachiereies. Anat. Anz. VI. 1891.

3) Rückert, J., Über physiologische Polyspermie bei meroblastischen Wirbelthiereiern. Anat. Anz. VII. Bd. 1892. 
die Konsequenz dieser Auffassung gegentuber seinen früheren Angaben über die Betheiligung der Merocyten zu der Bildung des Embryos; denn wir würden ja zu einer sehr abenteuerlichen Entwickelungstheorie gelangen, wollte er dieselben aufrecht erhalten. Er nimmt diese Angaben daher zurïck, glaubt aber an eine Einwanderung von Abkömmlingen der Merocyten in die Keimblätter, wo dieselben jedoch zu Grunde gehen sollen.

Zu erwähnen sind noch die Angaben von H. E. und F. ZIEGLER ${ }^{1}$ ), welche in den von ihnen untersuchten Stadien (vom sogen. Gastrulastadium $a b$ ) eine Betheiligung der Dotterkerne am Aufbau des Embryo leugnen. In einer späteren Arbeit ${ }^{2}$ ) bezweifelt H. E. ZIEgler die Ansicht RückenT's über den Ursprung der Dotterkerne und neigt sich der Ansicht von Kastschenko zu. Im Gegensatze dazu kommt LWOFF $^{3}$ ) auf die erste Arbeit RüCKERT's zurück, lässt das gesammte Entoderm und einen Theil des Mesoderms aus den Merocyten entstehen, während der Rest des Mesoderms und die Chorda ektodermalen Ursprungs sein soll; er selbst hat übrigens nur spätere Stadien untersucht.

Schließlich bemerke ich noch, dass Todaro ${ }^{4}$, ohne selbst Selachier untersucht zu haben, aus theoretischen Grïnden die Ansicht RückERT's von der Entstehung der Dotterkerne aus iberschüissigen Samenfäden angriff. Fr selbst hatte bei Seps, einer Echse, ursprünglich gemeint Polyspermie beobachtet zu haben, überzeugte sich aber später, dass das, was er zuerst für umgewandelte Samenfäden hielt, eigenthümliche, knötchenförmige Protoplasmaansammlungen (nodi protoplasmatici) sind. Da er in Folge dessen das Vorkommen physiologischer Polyspermie bei Reptilien zurïckweist, glaubt er diese Verneinung auch auf Selachier ausdehnen zu können. Da Herr Prof. Todaro die Freundlichkeit hatte, mir seine Präparate zu demonstriren, so will ich gern bestätigen, dass die von ihm gefundenen Nodi protoplasmatici nicht ron Samenfäden herrihhren kömnen, und ich glaube auch, dass bei Seps physiologische Polyspermie nicht vorkomme. Ich bestreite aber Todako jede Kompetenz, von diesem Befunde aus irgend einen Schluss auf die Verhält-

1) H. E. u. F. ZregLer, Beiträge zur Entwickelungsgeschichte von Torpedo. Arch. f. mikr. Anat. XXXIX. Bd. 1892.

2) Zieglen, H. E., Über das Verhalten der Kerne im Dotter der meroblastischen Wirbelthiere. Berichte d. Nat. Ges. z. Freiburg i. B. VIII. Bd. 1894.

3) 1. c.

4) Tobaro, F., Sopra lo sviluppo della Seps Chalcides, Ricerche f. n. Laboratorio di Anat. norm. d. Univ. Rom. Vol. III. 
nisse der Selachier zu ziehen, da bei diesen die Dottermasse in einer ganz anderen phylogenetischen Reihe erworben wurde, wie bei Reptilien.

Ich gehe nunmehr zu meinen eigenen Beobachtungen uiber and will vorausschicken, dass ich zwar glaube in einigen Punkten, so. bezüglich der Formen, Theilungsverhältnisse u. A. weiter gekommen zu sein, als die bisherigen Darstellungen; eine erschöpfende Darstellung der Formverhältnisse der Dotterkerne lag aber nicht in meiner Absicht; ich suchte mir bloB aus eigener Anschaunng eine Meinung ïber das Verhältnis derselben zu den Keimblättern zu bilden. So möchte ich insbesondere eine Frage gleich hier erledigen: ich spreche im Folgenden immer von den Dotterkernen; nun findet man häufig, aber nicht immer, um dieselben herum eine fein granulirte Masse, die man gewiss als den Zellleib, der zu dem Kerne gehört, ansehen kann; man könnte also eben so gut von Dotterzellen reden; wenn, wie ich glaube, die Ansicht RücKerT's über den Ursprung der Merocyten richtig ist, so würde das auch diese Auffassung stützen. Nun wäre es ja vielleicht möglich, den Zellleib auch in den Fällen, wo er nicht zu sehen ist, mit besonderen Methoden sichtbar zu machen; dessgleichen wäre es nicht ohne Interesse, die Veränderungen, die derselbe während der Umgestaltung: der Kerne erfährt, zu verfolgen. Für unsere Frage sind diese Dinge aber gleichgïltig; ich werde mich daher im Folgenden wesentlieh an die Kerne halten. Bemerken möchte ich noch, dass ich an die Existenz des von Scmultz und BaLfour beschriebenen Netz-oder wobl besser Fachwerks im Dotter nicht glaube; ich muss es mir aber versagen, darauf einzugehen, um nicht von meinem Thema allzusehr abzukommen.

Bezüglich des Ursprungs der Dotterkerne kann ich leider die schönen Untersuchungen RückERT's aus Mangel an Material in keiner Weise weiterführen; ich besitze nur zwei Keimseheiben, die ich mit einiger Wahrscheinlichkeit als Befruchtungsstadien deuten kann. Ich habe dieselben nicht selbst dem Mutterthier entnommen, sondern sie wurden mir in der zoologischen Station zu Neapel mit einem Stiuck des herausgeschnittenen Uterus übergeben. Da in jedem Falle nur ein Ei vorhanden war, während nach RüCKERT jedes Thier in der Regel sechs bis acht Eier enthält, so mahnt dies natiurlich zu Vorsicht. Da ich Hoffnung habe, geeigneteres Material noch zu erhalten, so würde ich den Befund an diesen Keimscheiben nicht veröffentlichen, wenn derselbe nicht in guter Übereinstimmung mit den Beobachtungen RückER's's stiinde.

Beide Keimscheiben stammen von Scyllium canicula; diejenige, 
die ich für jünger halte, enthält 40 Kerne, welche alle ziemlich gleichen Charakter haben (Fig. 2); sie befinden sich sämmtlich im Ruhestadium und besitzen ein von Chromatinkörnchen durchsetztes Gerüstwerk; die Weite der Hoblräume derselben sehwankt, so dass einzelne Kerne intensiver gefärbt erscheinen als andere. An der Oberläche der Keimscheibe finden sich zwei Körper, die zweifellos als Richtungskörper anzusprechen sind (Fig. 1). Das Vorhandensein derselben ist zwar an und für sich kein Beweis dafür, dass wir es mit einem sehr jungen Stadium zu thun haben; denn wir wissen durch die Untersuchungen von Kastschenko ${ }^{1}$ ), dass dieselben auch noch in viel späteren Stadien zu sehen sind. Ich selbst fand sie noch im vierzelligen (Fig. 20). Sie machen dort aber entschieden bereits den. Eindruck einer beginnenden Degeneration; es ist insbesondere der helle Kernraum um das Chromatin herum geschwunden und das Chromatin im zweiten Richtungskörper bereits abgeblasst.

Ich nehme an, dass sich unter den 40 beobachteten Kernen der männliche und der weibliche Vorkern befinden, während der Rest zu Dotterkernen wird. Die Vorkerne zu erkennen, ist mir freilich nicht gelungen; möglicher Weise könnte man zwei in der Mitte der Keimscheibe in geringer Entfernung von einander liegende Kerne dafür halten; allerdings haben beide ganz dasselbe Aussehen, während sich nach RückEnT der männliche Vorkern rom weiblichen deutlich unterscheiden soll. Eben so macht die große Zahl der Dotterkerne mich bedenklich, da RückERT, so weit er darüber Angaben macht, immer viel weniger gefunden hat; außerdem habe ich in späteren Stadien mitunter weniger Dotterkerne gefunden. Andererseits ist sicher, dass die Zahl derselben sehr variirt, und ich bleibe auch desshalb bei der gegebenen Deutung, da man sonst nur annehmen könnte, die Keimscheibe stelle überhaupt einen pathologischen Fall vor. Ein Furchungsstadium ist sie jedenfalls nicht; das Fehlen der Furchen beweist das zwar nicht, da dieselben auch im vierzelligen Stadium noch fehlen können; hingegen konnte ich in allen Fällen, wie aus dem Folgenden noch hervorgehen wird, mit großer Sicherheit die Furchungskerne von den Dotterkernen unterscheiden, wäbrend hier alle Kerne den gleichen Charakter haben.

1) Kastranenko, N., Über den Reifungsprocess des Selachiereies. Zeitsehr. f. wiss. Zool. Bd. L. 1890. Ich fand, wie aus meinen Zeichnungen hervorgeht; besonders was das Chromatin betrifft, die Verhältnisse etwas anders, als Kastschenko sie darstellt; da aber Kastschenko ein viel größeres Material zur Verfügung hatte, überdies in vielen Punkten große Variationen feststellen konnte, so will ich auf diesen Punkt nicht weiter eingehen. 
Für etwas älter halte ich die zweite Keimscheibe; sie besitzt Kerne, welche ganz ähnlich gebaut sind, wie die der eben beschriebenen. Hingegen finden sich hier in der Mitte der Keimscheibe zwei sich berihhrende Kerne (Fig. 3 und 4); sie sind in Bau und Größe völlig gleich ${ }^{1}$ ); ich halte sie für die in Vereinigung begriffenen beiden Vorkerne; welches der männliche und welches der weibliche ist, kann man freilich auch hier nicht unterscheiden. Fig. 5 stellt einen Dotterkern dar, der die große Ähnlichkeit mit den Vorkernen erkennen lässt. Richtungskörper habe ich an dieser Keimscheibe nicht angetroffen; ich habe solche aber überhaupt nur in den zwei bereits erwähnten Fällen gefunden, was ja mit den Beobachtungen KASTSCHENKo's ganz gut ibereinstimmt. Ich bemerke noch, dass die Deutung, die beiden einander anliegenden Kerne seien die beiden ersten Furchungskerne, die sich eben getheilt haben, entschieden auszuschließen ist, weil in diesem Fall die Kerne viel weiter aus einander liegen müssten. So bleibt auch hier nur die gemachte Annahme, oder die eines völlig pathologischen Verhaltens übrig.

Ein Stadium mit einem Furchungskern ist mir nicht zu Gesicht gekommen. Hingegen besitze ich eines mit zwei Furchnngskernen von Pristiurus, ferner im vierzelligen Stadium vier Keimscheiben von Scyllium canicula und eine von Raja. Ich will beide Stadien zusammen besprechen, da es ja hauptsächlich auf die Unterscheidung der Furchungskerne von den Dotterkernen ankommt. Bei der Keimscheibe im zweizelligen Stadium ist die Furche eben angedeutet, im vierzelligen Stadium fehlt jede Furche an einer Keimscheibe von Scyllium und der von Raja; bei Torpedo soll nach Rücken'r ein derartiges Verhalten noch viel häufiger und auch noch auf späteren Stadien vorkommen. Die Zahl der Dotterkerne beträgt in dem zweizelligen Stadium 31, im vierzelligen Stadium bei Scyllium 91, 65, 56 und 15, letzteres bei der Keimscheibe obne Furchen, bei Raja 41; ohne Weiteres fällt hier die große Verschiedenheit in der Zahl derselben auf, worauf schon von KASTSCHENKo und RüCKERT aufmerksam gemacht wurde.

In allen Fällen ließen sich, auch abgesehen von der Lage, die Furchungskerne von den Dotterkernen gut unterscheiden, und ich will daher die verschiedenen Zustände, in denen ich beide getroffen

1) Auf der Zeichnung (Fig. 4) ist der tieferliegende Kern (rechts) etwas kleiner; dies ruihrt aber daher; dass er erst im nächsten Schnitt voll getroffen wird. 
habe, beschreiben; ich nehme zunächst die Keimscheibe von Raja aus, da dieselbe so eigenthümliche Verhältnisse darbietet, dass ich dieselben im Zusammenhange besprechen muss. - Ich beginne mit dem Furchungskerne; da in jeder Keimscheibe sich alle Kerne im gleichen Stadium befinden, so sind mir natürlich nicht alle Phasen der Kerntheilung zu Gesicht gekommen. Einen Kern in Ruhe sehen wir in Fig. 21; derselbe sieht gleichmäßig granulirt aus und ist verhältnismäßig schwach gefärbt. Fig. 17 zeigt einen Kern im Knäuelstadium, dessen chromatischer Faden sebr fein erscheint. Weiter fortgeschritten ist der Kern in Fig. 8, wo die Chromosomen sich bereits gesondert haben; dieselben sind von beträchtlicher Dicke. Hieran schließt sich das Stadium des Muttersterns, das ich bei Raja gẹtroffen habe (Fig. 24); dasselbe bietet uns nichts Auffälliges, wird uns aber noch zum Vergleich mit den mitotischen Figuren der Dotterkerne von Werth sein. Von den Anaphasen kann ich nur ein Stadium wiedergeben und kann auch dieses nur mit einiger Wahrscheinlichkeit dahin rechnen. Es sind dies die Kerne des zweizelligen Stadiums, von denen ich annehme, dass sie sich in Rekonstruktion befinden (Fig. 6); auffallend ist hier der helle, fein granulirte Raum, der das Chromatin umgiebt und ron den Dotterkörnchen scheidet; ich glaube, dass derselbe bei der weiteren Ausbildung des Kernes in denselben einbezogen wird.

Von den Dotterkernen muss man annehmen, dass sie seit dem Befunchtungsstadium einige Veränderungen erlitten haben; denn man trifft sie nur ganz ausnahmsweise in der dort geschilderten Größe und mit einem von Vacuolen durchsetzten Kerngerüst; meist stellen sie wenig umfangreiche, oft ganz homogene, sehr intensiv färbbare Chromatinanhäufungen dar, die häufig aus einer Anzahl von einzelnen Körnern bestehen. Ein Übergangsstadium vom ersten zum zweiten Zustand stellt vielleicht Fig. 7 dar, welche einen Dotterkern des zweizelligen Stadiums wiedergiebt, an dem sich bereits eine größere Koncentration des Chromatins in Vergleich z. B. zu Fig. 5 zu erkennen giebt. Die Form der Dotterkerne kann sehr wechseIn: Fig. 22 und 23 zeigt stabförmige, geschwungene und ovale Formen, an denen die Chromatinmasse ganz homogen erscheint. In Fig. 18 und 19 sind welche zu sehen, welche mehr oder weniger rund und fein granulirt sind; schließlich bietet Fig. 16 einen Fall, der sehr häufig ist, in dem der Kern aus einer Anzahl von Chromatinkörnern besteht, die locker beisammenliegen. Alle diese Formen halte ich für ruhende Kerne. Die Theilung der Dotterkerne ist in diesen frïhen 
Stadien zweifellos meistens mitotisch; doch kommt sicher auch direkte Theilung vor. Auch die Karyokinese hat große Neigung: zu UnregelmäBigkeiten und Abweichungen. Ein Knäuelstadium habe ich nie gefunden, überhaupt nichts was sich als eine dem entsprechende Prophase deuten ließe. In den Fällen, wo der Kern im Ruhestadium kompakt ist, zerfällt er wahrscheinlich als Vorbereitung zur Theilung direkt in eine Anzahl von Chromosomen. An den Theilungsfiguren im Stadium des Muttersterns (Fig. 9, 10) fällt vor Allem auf, dass die Chromosomen nicht in Form einer regelmäßigen Platte, sondern ziemlich unregelmäßig zwischen den kegelförmigen Strahlenfiguren liegen; dessgleichen wechselt die Zahl der Chromosomen, wie schon ein Vergleich zwischen Fig. 9 und 10 ergiebt. Über das Vorhandensein von Centrosomen kann ich mich nicht äußern, da die von mir mit Vorliebe angewandte Färbung. (Boraxkarmin und Pikrinsäure) offenbar wenig geeignet ist, dieselben hervortreten zu lassen. Betrachten wir Theilungsfiguren im Stadium des Tochtersterns (Fig. 11, 12, 13), so zeigt sich in Fig. 12 und 13 sofort, dass die Vertheilung der Chromatimmenge auf die beiden Tochterkerne eine sehr ungleiche ist. Dasselbe ist auch der Fall an Fig. 14, welche ein weiter vorgeschrittenes Theilungsstadium zeigt, in dem die Verbindungsfäden der Tochterkerne in der Mitto der Durchschnürung nahe sind. In allen geschilderten Fällen sind die Chromosomen kugelförmig und nur hier und da findet sich eine Andeutung einer kommaförmigen oder mehr gestreckten Form; jedenfalls sind sie viel gröBer, als die Chromosomen der Furchungszellen, die überdies ausnahmslos schleifenförmig sind. Tch halte es daher für ganz ausgeschlossen, dass man einen Dotterkern in Theilung mit einem Furchungskern in der gleichen Phase verwechseln könnte; im Ruhestadium unterscheiden sich aber die Furchungskerne mindestens schon durch die Größe. Freilich muss ich bemerken, dass die geschilderten Theilungsfiguren alle von einer Keimscheibe von Scyllium im vierzelligen Stadium stammen, an der die meisten Dotterkerne in Theilung waren; in den andern Keimscheiben habe ich nur ganz vereinzelt Theilungen gefunden; es macht den Eindruck, als ob die Theilungen in den ersten Stadien annähernd synchron verliefen. Ich will daher meine Angaben uiber den Charakter der Theilungsfiguren nicht zu sehr verallgemeinem, um so mehr als ich auf einem späteren Stadium Theilungsfiguren beschreiben werde (Fig. 42), die ein anderes Aussehen haben. Dieselben erinnern an die Befunde RüCKERT's bei Torpedo, wo ein 
Unterschied zwischen. Furchungskernen und Dotterkernen in Bezug. anf die Regelmäßigkeit der Figuren nicht herrscht; hingegen besitzen die Dotterkerne nach RÜCKERT hier nur halb so viel Chromosomen wie die Furchungskerne. Auch in diesem Falle ergiebt sich natiirlich leicht eine Unterscheidung der Dotterkerne von den Furchungskernen.

SchlieBlich möchte ich noch auf Fig. 15, 18 und 19 hinweisen, welche furr eine direkte Kerntheilung sprechen. In Fig. 15 sehen wir zwei neben einander liegende, ungleich große Haufen ron Chromatinkörnern, die nur mehr durch ein Chromatinkorn mit einander verbunden sind; da jede achromatische Figur fehlt, so kann man wohl .eine direkte Kerntheilung annehmen. Eben so in Fig. 18 und 19, wo die Kerne so nahe beisammen liegen, dass sie unmöglich aus einer mitotischen Theilung hervorgegangen sein können (man vergl. z. B. den Abstand in Fig. 14). Tch nehme ïbrigens um so weniger Anstand, in diesen Fällen eine direkte Theilung anzunehmen, als wir noch sehen werden, dass in späteren. Stadien die mitotische Theilung gegen die direkte immer mehr zuriicktritt.

Eine gesonderte Besprechung erfordert, wie schon bemerkt, das vierzellige Stadium ron Raja. Es finden sich keine Furchen; die Furchungskerne bieten nichts Auffälliges, sie befinden sich alle im Stadium des Muttersterns (Fig. 24). Zu erwähnen wäre höchstens, dass die Achsen der beiden Spindeln, die man in Seitenansicht zu sehen bekommt, nicht parallel zur Oberfläche liegen, sondern in einem Winkel ron ca. $45^{\circ}$. Eigenthümlich ist jedoch das Verhalten der Dotterkerne; dieselben (Fig. 25, 26) sind sämmtlich rund, ziemlich grob und fein granulirt; eine weitere Struktur lässt sich nicht erkennen; einige besitzen eine Vacuole (Fig. 26). Sie sind stets von einer umfangreichen Protoplasmamasse umgeben, die ihre Fortsätze zwischen die Dotterkörner sendet. Das Merkwürdige ist nun, dass die Färbbarkeit dieser Kerne mit Boraxkarmin außerordentlich wechselt; während einige Kerne ziemlich intensiv rosa gefärbt sind, sind andere blasser als das sie umgebende Protoplasma, und lassen sich nur durch dieses erkennen; dass es sich dabei nicht etwa um eine missglückte Färbung handelt, geht daraus hervor, dass die Chromosomen in den Furchungskernen sehr intensiv gefärbt sind. Von dem geschilderten Verhalten muss ich noch zwei Ausnahmen feststellen; in dem einen Falle (Fig. 27) finden sich innerhalb einer Protoplasmamasse zwei Kerne, welche bedentend kleiner sind, als die vorhin beschrie- 
benen; in jedem findet sich ein kleiner, sehr intensiv gefärbter Nucleinkörper innerhalb eines ganz strukturlosen Raumes. Ein ähnliches Bild bietet Fig. 28, nur dass sich hier statt zweier drei Kerne vorfinden, deren Chromatin nicht kugelig; sondern fadenförmig ist; die Masse des Chromatins in jedem Kerne scheint hier noch geringfügiger zu sein, als im ersten Falle. Es lässt sich mit großer Wahrscheinlichkeit annehmen, dass in beiden Fällen die Kerne eben aus einer Theilung hervorgegangen sind; Zahl und Anordnung: der Kerne in Fig. 28 lässt auf eine direkte Theilung schließen. Welche Bedeutung diese von Scyllium abweichenden Verhältnisse bei Raja haben, lässt sich natürlich aus dem einen Falle nicht beurtheilen; ich möchte nur darauf hinweisen, dass hier die Unterscheidung zwischen Dotterkernen und Furchungskernen möglicherweise nicht so einfach wäre, wenn letztere sich nicht gerade in Mitose befänden.

Vom achtzelligen Stadium ron Scyllium catulus gebe ich in Fig. 29 eine Oberflächenansicht wieder. RückeRT bildet dasselbe Stadium von Scyllium ab, wo die Verhältnisse aber etwas andere sind, indem eine centrale Zelle von den übrigen sieben umgeben wird. An Fig. 29 lässt sich eine gewisse Symmetrie der Zellen zu der durch den Pfeil angedeuteten Ebene erkennen. Ob diese aber der Ebene der ersten Furche entspricht, lasse ich dahingestellt; um so mehr als schon im vierzelligen Stadium nach meinen Beobachtungen die Furchen nicht senkrecht auf einander stehen, wie BALFouR dies abbildet, sondern gebrochene Linien darstellen. $\mathrm{Zu}$ bemerken ist noch, dass die Zellen in der Mitte eine Luicke freilassen (Fig. $29^{*}$ ), in der der ungefurchte Dotter zu Tage tritt.

Am Oberflächenbild konnte ich von abgefurchten Merocyten, wie sie RücKERT von seiner Keimscheibe beschreibt, nichts bemerken; die Schnittserie, die ich von dieser Keimscheibe anfertigte, ist mir leider verunglückt.

Ein sechzehnzelliges Stadium von Scyllium canicula zeigt in der Oberflächenansicht bloß 14 Zellen; zwei Zellen stoßen, wie die Schnitte lehren, bloß mit einer Kante an die Oberfläche. Aus Fig. 30 ist zu ersehen, dass sigh die Zellen in Bezug auf ihre Abgrenzung gegen den Dotter sehr verschieden verhalten; während einige schon ganz von demselben abgegrenzt sind, gehen andere mit ihrer ganzen Basis in denselben uiber. Die Kerne der Furchungszellen befinden sich alle im Stadium der Äquatorialplatte; in drei Zellen stehen die Theilungsfiguren senkrecht zur Oberfläche, wäbrend 
die übrigen parallel oder nur wenig geneigt zu derselben liegen. RücKERT giebt an, dass spätestens nach dem 16zelligen Stadium ctwa ein Drittel der Zellen sich bei Torpedo nach der Tiefe zu theilt. Daraus geht hervor, dass ein Stadium, in dem alle Zellen sich nach der Tiefe zu theilen und das der Theilung durch Äquatorialfurchen bei holoblastischer Eiern entsprechen würde, bei Selachiern nicht vorkommt.

An Dotterkernen zähle ich in dieser Keimscheibe 163; sie zeigen sehr verschiedenes Aussehen; man findet rundliche (Fig. 31) und langgestreckte (Fig. 32) Formen, die gleichmäßig fein granulirt erscheinen; ferner solche, die eine kompakte, häufig gelappte Chromatinmasse darstellen (Fig. 33 und 34); ferner Formen, die aus einer Ansammlung von Chromatinkörnern verschiedener Größe bestehen (Fig. 35-37); einen in direkter Theilung begriffenen Kern zeigt Fig. 38.

Nach dem 16zelligen Stadium weist mein Material eine Lïcke auf, da das nächstältere Stadium, das ich besitze, bereits 79 Furchungszellen besitzt; daraus geht hervor, dass mindestens rom 64zelligen Stadium ab synchrone Theilungen nicht mehr erfolgen. Doch ist es wahrscheinlich, dass dieses vom 32zelligen Stadium ab nicht mehr der Fall ist; denn RückERr, der bei Torpedo noch ein Stadium von 128 Zellen angetroffen hat, giebt an, dass er bei Scyllium und Pristiurus bereits das 64zellige Stadium nicht mehr finden konnte. In der erwähnten Keimscheibe liegt ein Theil der Zellen bereits in zweiter Reihe; die an der Oberfläche liegenden überwiegen aber noch. Auffallend sind die Unterschiede in der Größe, die die in der Tiefe liegenden Zellen aufweisen, wie sich aus einem Vergleich des median geführten Schnittes der Fig. 39 und eines mehr lateralen (Fig. 40) ergiebt. In Bezug auf die Abgrenzung der Zellen gegen den Dotter herrscht noch große Verschiedenheit, indem sowohl von den in der Tiefe, als auch von den oberflächlich gelegenen Zellen einige bereits vollständig abgegrenzt sind, andere noch nicht. Zwischen den Zellen finden sich häufig Hohlräume und Spalten, die offenbar durch den Theilungsmechanismus extstanden und bei jeder neuen Theilung Veränderungen unterworfen sind und denen weiter keine Bedentung zukommt. Größeres Interesse dürfte eine helle Stelle unter den Furchungszellen, die man an Medianschnitten antrifft (Fig. 39), beanspruchen; hier fehlen die in der Umgebung iiberall dicht gelagerten Dotterkörner und an deren Stelle findet man einen blassen, feinkörnigen Niederschlag. Ich glaube, dass 
dies der Beginn der Bildung einer Höhle ist, die man in späteren Stadien regelmäBig vorfindet und die bisher von den Autoren als Furchungshöhle bezeichnet wurde. Nur persistirt diese Höhle in diesem Stadium noch nicht, sondern sie wird wieder ron den neugebildeten Zellen verdrängt; diese Annahme stimmt mit den Befunden an späteren Stadien sehr gut überein, wo die Furchungszellen oberff̈̈chlich keineswegs weiter greifen, gegen den Dotter zu aber wesentlich tiefer reichen.

Dotterkerne finden sich in dieser Keimscheibe etwa 184; unter denselben sind alle die Formen vertreten, die ich vom 16xelligen Stadium beschrieben habe. Es gelingt auch hier, immer die Dotterkerne von den Furchungskernen zu unterseheiden. Die Größe bietet jetzt zwar keine unterscheidenden Merkmale mehr, da die Kerne der Furchungszellen natürlich gegen frühere Stadien wesentlich kleiner geworden sind. Die Furchungskerne sind aber in dieser Keimscheibe immer blasser, in ihrer ganzen Substanz gleichmäßiger gefärbt als die Dotterkerne, und haben immer eine ganz regelmäBige runde Form; so dass selbst in Fällen, wo Dotterkerne und Furchungskerne ganz nahe beisammenliegen und die Zellen der letzteren vom Dotter noch nicht abgegrenzt sind, wie z. B. in Fig. 41, eine Unterscheidung der Kerne trotzdem nicht schwer fällt. Einige Dotterkerne befinden sich in Mitose; sie haben zum Theil ein anderes Aussehen als die vom 4zelligen Stadium beschriebenen. So sieht in Fig. 42 die Theilungsfigur sehr regelmäßig aus und die Chromosomen sind fein und schleifenförmig. Vergleicht man aber damit einen Furchungskern in derselben Theilungsphase (Fig. 43), so sieht man, dass die Masse des Chromatins im Dotterkern viel geringer ist als die des Furchungskernes und vielleicht nicht einmal die Hälfte des letzteren beträgt, so dass auch hier eine Unterscheidung nicht schwer fällt. Dies Verhalten ist also dem von RücKERT für Torpedo beschriebenen ganz ähnlich, worauf ich schon hingewiesen habe. Außer diesen regelmäßigen Theilungsfiguren finden sich aber auch wieder unregelmäBige, wie Fig. 44 beweist, die sich den vorhin beschriebenen mehr anschließen.

Während des weiteren Verlaufs der Furchung behalten die Dotterkerne zunächst die beschriebene Gestalt bei; die Furchungszellen rücken in dem Maße als sie sich weiter vermehren mehr in die Tiefe, und während die Zahl der oberflächlich gelegenen Zellen Anfangrs überwog, kehrt sich dieses Verhältnis im weiteren Verlauf der Furchung natuirlich um. In der Tiefe findet zuerst die Ab- 
grenzung aller Zellen gegen den Dotter statt, später an den Rändern der Keimscheibe an der Oberfläche. In Fig. 45 sehen wir unter der Masse der Furchungszellen zwischen diesen und dem Dotter eine Höhle, welche der Furchungshöhle BaLfoun's und RückerT's entspricht; man findet sie mitunter schon in früheren Stadien, auch excentrisch gelegen, sie kann aber auch auf späteren Stadien noch fehlen. Auf den Namen Furchungshöhle hat dieser Hohlraum meines Erachtens keinen Anspruch; denn damit bezeichnet man bei holoblastischen Eiern den zwischen den Furchungszellen, besonders im Blastulastadium befindlichen Hohlraum; die Höble aber, um die es sich hier handelt, liegt gar nicht zwischen den Furchungszellen, sondern unter denselben; die Bezeichnung ist allerdings ganz korrekt, wenn man den Dotter und die Dotterkerne als einen Theil des Entoderms ansieht (BALFour, RücKeRT [erste Arbeit], LwOFF); wir werden aber sehen, dass diese Auffassung unhaltbar ist. Statt des Ausdrucks Furchungshöhle wäre der Name "Subgerminalhöhle« wohl angebrachter; doch wird letzterer Ausdruck bei Amnioten für ein Gebilde angewendet, dem vielleicht eine andere Bedeutung zukommt. Ich möchte daher den Ausdruck "Resorptionshöhle anwenden, der lediglich an die physiologische Bedeutung dieser Bildung ankaüpft; dass diese wirklich darin besteht, die vom Dotter unter dem Einfluss der Dotterkerne gebildete Nährfiüssigkeit aufzunehmen und an die Furchungszellen weiter zu geben, ist mir nicht zweifelhaft; wir sehen auch in Fig. 45 am Boden der Furchungshöhle einen fein granulirten Niederschlag $\left(^{*}\right)$, der wohl auf die Fällung einer eiweiBhaltigen Flüssigkeit zurückzuführen ist.

In Fig. 45 sieht man ferner, dass die Furchungszellen iiberall gegen den Dotter scharf abgegrenzt sind; dies ist aber nicht an allen Schnitten der Fall; an einem mehr lateral geführten Schnitt (Fig. 46) sieht man, dass die Abgrenzung einer oberflächlich gelegenen Zelle unvollständig, die einer andern noch gar nicht erfolgt ist; trotzdem kann man ebenso wie in früheren Stadien den Kern der letzteren von den Dotterkernen ganz gut unterscheiden. Es ist dies übrigens die älteste, mir zu Gesicht gekommene Keimscheibe, an der die Abgrenzung der Furchungszellen vom Dotter noch nicht völlig vollzogen ist; andererseits habe ich jüngere Stadien geseher, bei denen dies bereits der Fall war. Die Dotterkerne sind in dieser Keimscheibe größtentheils noch in derselben Form vorhanden, wie in den früheren Stadien; einige zeigen aber bereits jene Umwandlungen, die schließlich von allen durchgemacht werden: sie 
werden groß, bläschenförmig und zeigen ein Kerngerüst, das von bald größeren, bald kleineren Vacuolen durchsetzt ist; zugleich zeigen sich Bilder einer lebhaften direkten Kerntheilung. Diese Veränderungen will ich aber lieber am nächsten Stadium beschreiben, wo die meisten Kerne bereits von diesen Veränderungen betroffen sind.

Einen Durchschnitt durch diéses Stadium giebt Fig. 47 wieder; dass es beträchtlich älter ist, als das der Fig. 45, ergiebt schon ein Vergleich der Größe der Furchungszellen. Von den Dotterkernen hat nur mehr ein geringer Theil kompakte Form, oder besteht aus einer Anhäufung von Chromosomen; die Mehrzahl stellt große Kerne mit schwammigem Gerüst vor, die häufig in Zwei- bis Vierzahl beisammenliegen. Dieser letztere Zustand wird, wie ich vermuthe, auf folgendem Wege erreicht: ein Dotterkern, der aus einer Anzahl bald gröberer, bald feinerer Chromatinbrocken besteht, zerlegt sich in mehrere, meist ungleiche Chromatinhaufen (Fig. 48); bei kompakten Kernen dürfte die Theilung in Chromatinstücke diesem Stadium vorausgehen. Nunmehr sondert sich um das Chromatin herum ein heller Raum, der durch eine Membran von den Dotterkörnern abgegrenzt wird (Fig. 49); darauf treten auch zwischen den Chromatinhaufen Scheidewände auf (Fig. 50, 51) und dadurch sind die neuen Kerne gebildet; sie treten aus einander und runden sich ab. Gleichzeitig lockert sich die Chromatinmasse und wird zackig; wodurch der freie Raum um dasselbe herum allmählich verdrängt wird (Fig. 52, 53); die Endstadien sehen wir in Fig. 54-56, wo wir mehrere Kerne dicht beisammen liegend finden, die ein von Vacuolen durchsetztes Gerüst aufweisen; in manchen Kernen findet man auch fädige Struktur. In Bezug auf die Intensität der Färbung variiren die Kerne sehr, was aber gar nicht Wunder nehmen kann, da wir ja gesehen haben, wie ungleich die Chromatinmasse auf die Kerne vertheilt wird. Man findet übrigens Kerne in den verschiedensten Größen; ganz kleine Kerne, die man gelegentlich antrifft, stammen möglicherweise von Chromatinpartikeln ab, die, wie in Fig. 52 zu sehen ist, bei der Theilung übrig bleiben, oder auf andere Weise versprengt werden. Außer den direkten Theilungen findet man auch Mitosen, wenn auch spärlich; es ist dieses das älteste Stadium, auf dem ich noch welche getroffen habe. Die Theilungsfiguren stimmen mit denen im vierzelligen Stadium überein, haben grobe, kugelige Chromosomen und琵zeigen häufig UnregelmäBigkeiten. Ich bilde eine solche in Fig. $57 \mathrm{ab}$, wo die Chromosomen der Tochterkerne sich eben getrennt haben, die Achsen, der achromatischen kegelförmigen Figuren aber einen Winkel bilden. 
Die weiteren Veränderungen der Furchungszellen, die bereits mit der Bildung der Keimblätter, zusammenhängen, werde ich im nächsten Abschnitt zu besprechen haben; hier will ich nur noch über die weiteren Schicksale der Dotterkerne Einiges bemerken. Sie verwandeln sich schließlich alle in die oben beschriebenen blasigen Formen; ein Theil der Kerne rückt an die Oberfläche des Dotters, wo sie sich mehr oder weniger abplatten; allmählich folgen dann die ibrigen Dotterkerne nach und spätestens in BaLFour's Stadium F liegen alle Kerne oberfächlich. Mittlerweile nehmen manche Kerne an Größe beträchtlich zu; ihr Gerüst bekommt ein verschwommenes Aussehen und das Chromatin beschränkt sich auf einige größere Kugeln, die dem Gerüstwerk eingelagert sind (Fig. 58, 59). Davon, dass diese Vergrößerung durch direkte Aufnahme von Dotterkörnern erfolgt, wie RückERT will, konnte ich mich nicht überzeugen; hingegen halte ich es für möglich, dass besonders große Kerne (wie Fig. 59) durch Zusammenfließen mehrerer Kerne entstehen. Später finden derartige Kernverschmelzungen zweifellos statt; wenn nämlich alle Kerne an der Oberfläche des Dotters liegen, dann sieht man bei Oberflächenansicht die Dotterkerne meistens sehr lange Streifen vorstellen. Ich bilde in Fig. 60 die Dotterkerne rom Dottersack eines $20 \mathrm{~mm}$ langen Embryos ab; ïber den Dotterkernen liegen natürlich noch die drei Keimblätter, die ich in der Zeichnung weggelassen habe; aus Fig. $60 \mathrm{a}$, wo ich den Umriss des mit * bezeichneten Dotterkerns der Fig. 60 bei derselben Vergrößerung gezeichnet habe, wie die Dotterkerne früherer Stadien, wird man entnehmen können, wie sehr die Größe der Dotterkerne zugenommen hat; zudem sind die in Fig. 60 abgebildeten Kernstreifen noch gar nicht die längsten; es giebt solche, die dreiund viermal so lang sind. Dabei hat die Zahl der Kerne gegen frühere Stadien sehr abgenommen, so dass mir die Annahme ganz ungezwungen scheint, dass mehrere Kerne sich zur Bildung der großen Kerne dieses Stadiums vereinigt haben.

Die Betheiligung von Merocyten am Aufbau des Embryos kann man auch für spätere Stadien mit großer Bestimmtheit ausschließen; ebenso konnte ich mich an meinem Material nicht davon überzeugen, dass einzelne Dotterkerne sich mit dem sie umgebenden Dotter abschnüren und in die Gewebe einwandern, um da zu Grunde zu gehen. Ich habe zwar an einigen, aber durchaus puicht an allen Keimscheiben, an denen das Ektoderm schon gebildet war, im Entoderm einige Zellen gefunden, deren Kern offenbar degenerirt war; 
Übergangsformen zu den Dotterkernen habe ich aber nicht gefunden. Desshalb will ich ihre Abstammung rom Dotter nicht mit Bestimmtheit in Abrede stellen; jedenfalls hat aber der Vorgang hier nur eine beschränkte physiologische Bedentung. Die bestimmten Angaben von RÜckerT ïber diesen Punkt will ich damit durchans nicht in Zweifel ziehen, ich glaube aber, dass dieselben hauptsächlich für Torpedo Guiltigkeit haben; bei Torpedo liegen die Verhältnisse in so fern ganz anders, als die Größe der Keimscheibe im Vergleich za der des Embryos viel geringer ist als bei Scyllium und Pristiurus. Da in Folge dessen die mit dem Dotter in Beriihrung: stehende, rosorbirende Fläche relativ klein ist, so tritt als Ersatz dafuir die Eimrichtung auf, dass der Dotter mit seinen Kernen in die Keimblitter einwandert, um da resorbirt zu werden.

Über das schlieBliche Schicksal der Dotterkerne habe ich keine Beobachtungen machen können, də mir dafür geeignetes Material nicht zur Verfügung stand. Da ich dieselben aber bis zu einem Stadium verfolgt habe, wo bereits alle Gewebe angelegt sind, und immer eine Betheiligung derselben am Aufbau des Embryos ausschließen konnte, so ist das auch fur spätere Stadien mit Sicherheit auszuschließen; es wäre auch nach ihrer Lage nnd Gestalt in dem letzten Stadium, in dem wir sie betrachtet haben (Fig. 60), ganz unmöglich. Ihr Schicksal kann daher meines Erachtens nur das sein, dass sie zu Grunde gehea, nachdem sio ihre Aufgabe, deu Dotter resorptionsfühig zu machen, erfiullt haben.

Wenn ich nun aus dem Vorstehenden das heransgreife, was für meine Aufgabe von besonderer Wichtigkeit ist, so muss ich erstens betonen, dass Alles, was ich gesehen habe, für die Annahme RÜckERT's spricht, dass die Dotterkerne nicht rom Furchungskerne abstammen, sondern wahrseheinlich von überschuissigen, in das Ei eingedrungenen Samenfäden; zweitens, dass die Dotterkerne am Aurbau des Embryos keinen Antheil haben. Neu sind diese Resultate ja nicht; denn im ersten Punkte konnte ich der Beweisfihhrung BückerT's nur wenig hinzufügen, im zweiten Punkte stimme ich mitC. K. HofFuAn, RüCKERT's jetzigem Standpunkte, sowie mit H.E. und F.ZIEGLER, die dasselbe fur die Stadien behaupten, die sie ans eigener Anschaung kennen, überein. Die Hauptsache für mich war aber, mir ein selbständiges Urtheil darüber zu bilden, welche Stellung den Dotterkernen den Keimblättern gegenüber zukommt; es ist nun ohne Weiteres klar, dass falls die Annahme RückEn's über die Herkunft der Dotterkerne richtig ist, diese mit den Keimblätteru 
uiberhaupt nichts zu thun haben. Sollte aber auch wider Erwarten diese Auffassung falsch sein, so müssten die Dotterkerne mindestens vom ersten Furchungskerne ihren Ursprung nehmen und hätten ron da ab mit den Furchungskernen gar keinen Zusammenhang mehr. Sie hätten also in diesem Falle schon einen ganz anderen Ursprung als die Makromeren holoblastischer Eier; überdies fände sich nicht der geringste Zusammenhang zwischen ihrer Entstehung und der des embryonalen Entoderms; dessgieichen nehmen sie an der Bildung der Keimblätter keinen Antheil. Sie dem Entoderm zuzurechnen und den Dotter der Selachier mit den Makromeren der Cyclostomen, Amphibien ete. za homologisiren, wäre daher durch nichts gerechtfertigt. Auch im Falle dieses Ursprungs der Dotterzellen könnte man in denselben nichts Anderes sehen, als die Abkömmlinge einer Furchungszelle, die sich der Funktion der Dotterresorption angepasst haben und nach erfullter Aufgabe zu Grunde gehen. Eine morphologisehe Bedeutung kommt ihnen auch in diesem Falle nicht zu und sie bleiben bei Beurtheilung der Keimblätterbildung außer Betracht. Eine dritte Möglichkeit ihrer Entstehung giebt es aber nicht.

\section{Die Bildung der primären Keimblätter.}

Man kann die Ansichten, welche beziiglich der Entodermbildung and Gastrulation bei Selachiern geäußert wurden, im Allgemeinen in zwei Gruppen eintheilen, je nachdem eine Einstülpung des gastralen Entodermsi) angenommen wird oder nicht.

Die letztere Anschaung wird vor Allem von BALFoun vertreten; dieser Forscher unterscheidet bereits in späteren Furchungsstadien eine oberflächliche Zellschicht als Ektoderm, während der Rest der Furchungszellen als Entoderm angesehen wird. Am Embryonaltheil des Keimscheibenrandes wölbt sich das Ektoderm dann etwas empor and im Anschluss an das Ektoderm reihen sich die Entodermzellen

1) Bezuiglich der Bezeichnungsart bemerke ich, dass man etwa von BALFouR's Stadium C an, sowohl nach Form als Bestimmung;, das Entoderm, das" in den Embryonalkörper übergeht (gastrales Entoderm;, von dem des Dottersackes (Dotterentoderm) scharf unterscheiden kaun; auch in früheren Stadien kann man wenigstens einen Theil des gastralen Entoderms mit Sicherheit als solches erkennen, wenn auch seine Abgrenzung gegen das Dotterentoderm eine unbestimmte ist; dem Ursprung nach sind aber beide Entodermantheile völlig gleich, wie ich im Gegensatz zu den meisten Antoren annehme. 
zu einem Cylinderepithel an einander. Ausdruicklich verwahrt sich Batfour gegen die Deutung; die HaEcker seinen Beobachtungen im Sinne einer Einstïlpung gab; es ist daher schwer verständlich, wie Kollmand ${ }^{1}$ ) dazu kommt, Balfour als Vertreter der'Einstülpungstheorie anzufubren. Als Gastrulation fasst BALFour das Einwachsen der Entodermzellen aus dem Dotter auf und die damit verbundene Verdrängung der Furchungshöhle; die ganze Peripherie der Keimscheibe ist nach seiner Auffassung Urmund.

In Bezug auf die Bildung der Keimblätter steht RücKERT in seiner ersten Arbeit gerade auf entgegengesetztem Standpunkte wie BALFour; während nach Letzterem bloß die oberste Schicht der Furchnngszellen zu Ektoderm wird, werden nach RückERT alle Furchungszellen zu Ektoderm, während das gesammte Entoderm von den Merocyten geliefert wird. Hingegen stimmt RückenT darin mit BALFơn überein, dass er gleichfalls eine Einstiulpung nicht annimmt; in seiner ersten Arbeit giebt er an, dass sich die von den Merocyten abgeschniurten Entodermzellen vom embryonalen Rande gegen die Mitte hin zu einem Epithel an einander reihen, wodurch eine "Pseudoinvagination" zu Stande kommt. In einer späteren Arbeit $\left.^{2}\right\rangle$ fasst er die Mechanik des Vorgangs etwas anders auf, er nimmt an, dass die Epithelbildung des Entoderms dadurch eingeleitet wird, dass am embryonalen Rande eine starke Abgabe ron Entodermzellen seitens der Merocyten stattfindet, wodurch das Blastoderm zuerst steil emporgerichtet und dann nach hinten umgefaltet wird; so dass, wenn ich RüCKEnT recht verstehe, der Rand der Keimscheibe zu Beginn dieses Vorganges als Anheftungsstelle des gastralen Entoderms bestehen bleibt, während sich das Blastoderm nach hinten zu weiter verschiebt. Ebenso wie BaLfour sieht RüCKkRT den ganzen Rand der Keimscheibe als Urmund an. Wie weit RÜCKERT gegenwärtig noch an diesen Ansichten festhält, ist aus seinen letzten. Veröffentlichungen nicht zu entnehmen; in ihrem vollen UTmfange kann er sie aber jetzt, wo er eine Betheiligung: der Merocyten am Aufbau der Keimblätter ausschließt, wohl nicht mehr aufrecht erhalten.

Weit verbreiteter als die eben erwähnte Ansicht ist die, dass das gastrale Entoderm durch Einstïlpung des Blastoderm-

1) Kolmmana, J., Gemeinsame Entwickelungsbahnen der Wirbelthiere. Arch. f. Anat. u. Entwickl. 1885.

2) RücknRT, J., Weitere Beiträge zur Keimblattbildnng bei Selachiern. Anat. Anz. IV, 1889. 
epithels entsteht; dieser Ansicht sind Haeckel ${ }^{1}$ ), Kastschenko ${ }^{2}$ ), H. E. und F. Ziegler ${ }^{2}$ ), Kollamand ${ }^{2}$ ), Rabl ${ }^{3}$ ), Perenyi ${ }^{4}$ ).

Von den meisten Anhängern der Einstiulpungstheorie wird der Keimscheibenrand in seinem ganzen Umfange als Urmund angesehen, von einigen aber nur der an der Einstülpungsöffnung gelegene Theil desselben. Welcher Meinung SchuLtz ${ }^{2}$ ) war, ist nicht genau zu entnehmen, doch scheint es, dass er wenigstens eine theilweise Einstülpung annahm; einen vermittelnden Standpunkt nimmt SWAEN ${ }^{2}$ ) ein, der das gastrale Entoderm von einer noch nicht in Keimblätter differenzirten Zellmasse am Rande der Keimscheibe herleitet.

Eine Einstuilpung des Blastodermepithels nimmt anch LwofF ${ }^{2}$ ) an; er fasst dasselbe als Ektoderm auf, welches die Chorda und einen Theil des Mesoderms liefern soll; dass alles Übrige von den Merocyten hergeleitet wird, habe ich im ersten Abschnitte bereits erwähnt.

Nach meinen Beobachtungen leitet sich die Keimblattbildung: damit ein, dass die Keimscheibe nach dem in Fig. 47 dargestellten Stadiun sich an der Oberfläche mehr ausbreitet und dabei an Tiefe abnimmt, wodurch auch in den Fällen, wo im vorigen Stadium keine Resorptioushöhle vorhanden war, eine solche entsteht, die ausuahmslos am embryonalen Ende der Keimscheibe gelegen ist (mit RüCKERT gegen BALFour). Mit dieser Ausbreitung, die auch während der folgenden Stadien fortschreitet, geht die Ausbildung des Ektoderms Hand in Hand, und zwar in der Weise, dass die oberflächlich gelegenen Schichten der Keimscheibe sich zu einem Epithel zusammenfuigen, welcher Vorgang vom embryonalen Rande der Keimscheibe aus allmählich fortschreitet. Die unteren Zellschichten, welche in die Bildung des Ektoderms nicht aufgegangen sind, bleiben zunächst in lockerem Verband unter dem Ektoderm liegen (Fig. 61); mitunter findet sich wie in Fig. 61 zwischen den beiden Keimblättern eine Höhle $\left(^{*}\right)$, die mit der Resorptionshöhle nicht zusammenhängt; sie könnte, da sie zwischen den primären Keimblättern liegt, als das Rudiment einer Furchungshöhle aufgefasst werden. Da sie aber in der Mehrzahl der Fälle fehlt, hat sie für die Keimblätterbildung. offenbar keine Bedentung. Sowohl das Ektoderm wie das Entoderm

1) Haeckel, E., Die Gastrula u. die Eifurchung der Thiere. Jen. Zeitschr. f. Nat. IX. Bd. 1875 .

3) $1 . \mathrm{c}$.

3) Rabl, C., Theorie des Mesoderms. Morphol. Jahrb. XV. Bd. 1889.

4) Perenyr, Embryologie von Torpedo. Zool. Anz. $18 \$ 6$. 
gehen in die noch undifferenzirte Masse der Furchungszellen iber; beim Ektoderm ist dieser Ühergang ein ganz allmählicher, indem das Epithel immer dicker und mehrschichtig wird, bis sehließlich die Zellen ïberhaupt nicht mehr epithelial angeordnet sind.

Nicht immer ist iibrigens auch in den frïhesten Stadien das Ektoderm vom Entoderm am embryonalen Keimscheibenrand so scharf abgesetzt, wie dies Fig. 61 zeigt; das epitheliale Ektoderm geht vielmehr meistens am Embryonalrande in einen Haufen unregelmäßig gelagerter Entodermzellen über, so wie wir es an einem etwas vorgeschritteneren Stadium in Fig. 62 sehen. Diese Keimseheibe ist gegen die der Fig. 61 nicht in dem Maße weiter entwickelt, als man nach der größeren Zahl der Zellen schließen müsste; es scheint, dass in dieser Beziehung größere Verschiedenheiten herrschen, indem bei Beginn der Keimblattbildung bald mehr, bald weniger Zellen vorhanden sein können. Die dichte Zusammenfügung der Entodermzellen, wie wir sie in Fig. 62 direkt am Rande antreffen, ist bereits die Einleitung zur Bildung des Entodermepithels; die aächsten Stadien dieses Processes zeigen Fig. 63 und 64. Wir können an denselben die fortschreitende Abhebung der in Fig. 62 dem Dotter noch mit der ganzen Basis aufliegenden Entodermzellen verfolgen; die Umbildung der zuerst unregelmäBig gelagerten Entodermzellen zu einem Cylinderepithel folgt diesem Process erst nach und erstreckt sich Anfangs noch gar nicht über den ganzen Bereich der vom Dotter abgehobenen Entodermzellen. Wir treffen also in diesem Stadium (Fig. 64) am Rande der Keimscheibe die Grenze zwischen Ektoderm und Entoderm; an der unteren Seite vom Rande gegen die Mitte zu fortschreitend das Entoderm zuerst auf eine kurze Strecke in Form eines Cylinderepithels, dann als kompakte Zellmasse, schließlich als lockeren Zellhaufen, der in die noch nicht differenzirten Furehungszellen übergeht. In allen diesen drei Theilen ist das Entoderm vom Ektoderm so sehr verschieden, dass man eine Entstehung des ersteren durch Einstïlpung aus dem Ektoderm unmöglich annehmen kann.

Die Differenzirung des Ektoderms schreitet im Allgemeinen vom embryonalen Rande aus gleichmäßig fort; in dem Maße, als das Ektodermepithel sich weiter bildet, sind auch die zwischen demselben und dem Dotter gelegenen Zellen als Entoderm zu betrachten. Auch das Entoderm fügt sich, wie wir bereits gesehen haben, vom embryonalen Rande aus zu einem Epithel zusammen; hier geht jedoch der Process viel langsamer vor sich als beim Ektoderm. Ich 
fand sogar in einem Falle an einer Keimscheibe bereits das ganze Ektoderm als Epithel vor, während das Entoderm noch einen darunterliegenden, ganz regellosen Zellhaufen vorstelite; ein derartiges Verhalten ist aber kein normales. Die Regel, dass die Differenzirung: rom Embryonalrande aus fortschreitet, erleidet mitunter Ausnahmen; so sehen wir, dass in Fig. 63 sich mitten im Haufen der undifferenzirten Furchungszellen auf eine Strecke die Keimblätter geschieden haben; zwischen denselben findet sich ein Hohlraum (*), den man ähnlich wie in Fig. 61 als rudimentäre Furchungshöhle ansehen kann. In ähnlicher Weise ist auch in Fig. 64 an dem dem embryonalen entgegengesetzten Rande ein Stück Ektoderm bereits differenzirt.

Hat sich das Ektoderm im ganzen Bereich der Keimscheibe als Epithel gesondert, so ist die Bildung der primären Keimblätter vollzogen, da alle zwischen dem Ektoderm und dem Dotter gelegenen Zellen als Entoderm zu betrachten sind; es eriibrigt noch, dass auch diese sich zu einem Epithel zusammenfügen. Dieser Process geht aber sehr langsam vor sich und gelangt erst zum Abschlusse, wenn anch schon das Mesoderm angelegt ist. An der Keimscheibe, ron der in Fig. 65-67 Sagittalschnitte dargestellt sind, sind die Keimblätter bereits völlig gesondert; das Entoderm ist aber nur auf eine kurze Strecke epithelförmig und vom Dotter abgehoben (Fig. 65); bemerkenswerth ist auch hier, dass das Entoderm dicker ist als das Ektoderm, sowie auch mehrschichtig und desshalb von letzterem ohne Weiteres leicht zu nnterscheiden. Im übrigen Bereich der Keimscheibe wird das Entoderm überall durch eine Schicht von lose beisammen liegenden, spindelförmigen, oder auch mit mehreren Fortsätzen versehenen Zellen dargestellt (vgl. den lateral gefübrten Schnitt Fig. 66); nur an den Rändern (Fig. 66 und 67*) sehen wir, dass die Entodermzellen beginnen, sich zu einem Plattenepithel zusammenzufügen; es scheint dies die Regel zu sein, dass die Epithelisirung des Entoderms rom ganzen Rande aus gegen die Mitte zu fortschreitet, so dass die, fuir das Ektoderm wenigstens im Allgemeinen geltende Regel, dass die Epithelbildung rom Embryonalrande gegen die Mitte zu fortschreitet, für das Entoderm beträchtlich modificirt ist.

Für die Frage, ob das gastrale Entoderm von der oberflächlichen Epithelschicht durch Einstiulpung gebildet wird oder nicht, ist eine Keimscheibe ron Interesse, die etwas älter ist, als die eben beschriebene; Fig. 68 zeigt ein Oberflächenbild des embryonalen 
Keimscheibenrandes, an dem sich die Partie, in der eine epitheliale Anordnung des Entoderms erfolgt ist, durch ibre dunklere Färbung leicht erkennen lässt; man sieht, dass dieselbe nicht etwas sichelförmig, sondern linsenförmig ist, dass also die Ansatzstelle des epithelialen Entoderms nicht konvex ist im Sinne des Keimscheibenrandes, sondern konkav. Querschnitte bestätigen diese Deutung. rollkommen; die ersten Schnitte der Serie zeigen beide Keimblätter in Epithelform und das Entoderm ist ganz vom Dotter abgehoben; weitere Schnitte zeigen das Entoderm in der Mitte epithelial und vom Dotter abgehoben, während es seitlich demselben aufliegt und aus unregelmäßig gelagerten, aber kontinuirlich zusammenhängenden Zellen besteht (Fig. 69); noch weiter vorn (Fig. 70) findet man das Entoderm in der Mitte des Schnittes der Embryonalachse entsprechend als solide mehrschichtige Zellmasse, die dem Dotter unmittelbar aufliegt; seitlich davon stellt es zum Theil schon ein Plattenepithel dar. Ein Schnitt durch die Mitte der.Keimscheibe endlich (Fig. 70) zeigt uns das Entoderm als eine Schicht regellos angeordneter, theils spindelförmiger, theils mit mehreren Fortsätzen versehener Zellen; in der Mitte des Schnittes sind dieselben am zahlreichsten.

Schon BaLfour hatte einen ähnlichen Schnitt, wie den der Fig. 69, gegen die Einstülpungstheorie ins Feld geführt. Die Anhänger derselben äußern sich ja im Allgemeinen wenig darüber, wie man sich diesen Einstülpungsprocess mechanisch vorstellen soll; sie dürften aber meist die von den Brüdern ZIEGLER ausgesprochene Ansicht hegen, dass das Blastoderm iiber seine Ansatzstelle am Dotter hinauswächst, wobei nach ZIEGLER die dem Blastodermrande anliegenden Dotterentodermzellen denselben gewissermaßen festhalten sollen; es wäre auch sehwer, sich die Sache irgendwie anders vorzustellen. SWAEN nimmt allerdings ein freies Hineinwachsen des von der Randzone gelieferten Entoderms in den Raum zwischen Ektoderm und Dotter an, aber seine Bilder, in denen man immer zwischen Entoderm und Dotter einen freien Raum und keinerlei Anheftungsstelle des epithelialen Entoderms findet, entsprechen offenbar nicht den Thatsachen. Wenn nun aber die Annahme der Brìder ZreGLen richtig wäre, so müsste wenigstens während der ersten Stadien der Einstuilpung die Anheftungsstelle des epithelialen Entoderms am Dotter dem Keimscheibenrand eines früheren Stadiums entsprechen; diese Linie müsste daher in demselben Sinne konvex sein wie der Keimscheibenrand, und der zwischen Entoderm und Dotter entstehende Raum müsste sichelförmig sein; an der beschriebenen 
Studien über den Einfluss des Dotters auf die Gastrulation etc. I. 161

Keimscheibe ist aber gerade das Gegentheil der Fall. Wie sehr auch alle anderen Befunde gegen eine Einstïlpung sprechen, habe ich bereits hervorgehoben.

Die weitere Ausbildung des Entoderms zeigen uns Sagittalschnitte durch ein Stadium, das etwa BaLfour's Stadium B entspricht. Schon bei Oberflächenbetrachtung der Keimscheibe fielen zwei große, von einandèr getrennte Resorptionshöhlen in der Mitte der Keimscheibe auf, die in Fig. 72 auf dem Durchschnitt zu sehen sind. So große Höhlen habe ich auf diesem Stadium nur in diesem Falle gesehen, kleinere dagegen sehr häufig und zwar auch immer in der Mitte oder nahe der Mitte; es scheint, dass durch das Wachsthum der Keimscheibe die ursprünglich am Rande gelegenen Höhlen nach der Mitte zu verlagert werden; es wäre aber auch nicht ausgeschlossen, dass es sich um Neubildungen handelt, da gerade die unmittelbar vorausgehenden Stadien diese Bildungen selten zeigen. Die Höhlen sind, wie Fig. 72 zeigt, sowohl gegen den Dotter als gegen das Entoderm durch eine Lage entodermalen Plattenepithels abgegrenzt. Auch im ïbrigen Bereich der Keimscheibe außerhalb des embryonalen Bezirkes hat sich das Entoderm zu. einem Plattenepithel umgestaltet. Gegenüber den Angaben der Brüder Zregler, dass bei Torpedo das Dotterentoderm vom Rande her gegen die Mitte zu vorwächst, möchte ich bemerken, dass bei Pristiurus und Scyllium zu einer derartigen Annahme kein Grund vorliegt; das Dotterentoderm entsteht hier wie das gesammte Entoderm an Ort und Stelle, indem es jenen Rest der Furchungszellen vorstellt, der in die Bildung des Ektodermepithels nicht mit eing'egangen ist. Im Bereich des gastralen Entoderms ist die Abhebung vom Dotter etwas fortgeschritten (Fig. 73), und der Spalt zwischen Entoderm and Dotter setzt sich eine Strecke weit zwischen die Zellen des Entoderms hinein fort. Dies ist aber nicht so aufufassen, als ob hier bereits der Beginn des ventralen Darmverschlussas vorläge, denn dieser beginnt erst in einem späteren Stadium, in dem bereits eine sehr'umfangreiche Darmhöhle vorliegt. Diese Fortsetzung des Spaltes zwischen die Entodermzellen findet sich, wenn auch nicht so ausgesprochen, schon auf früheren Stadien und hängt wahrscheịnlich mit Wachsthumsverhältnissen zusammen. Das Entoderm in Fig. 72 (Fig. 73 bei stärkerer Vergrößerunğ) entspricht gerade der Chordaanlage und es ist auch hier zu beachten, wie sehr das Entoderm durch seine Dicke und Mehrschichtigkeit gerade am Keimscheibenrande von dem dariiberliegenden Ektoderm verschieden 
ist; wie für die früheren Stadien, so ist anch für dieses jede Einstülpung des Ektoderms durch diesen Befund völlig auszuschließen. Wie in früheren Stadien, so schliebt sich auch in diesem an das epitheliale Entoderm die kompakte Masse desselben an, die in die locker beisammenliegenden Zellen allmählich übergeht (Fig. 73). An einem etwas lateral geführten Schnitt (Fig. 74) ist das Entoderm nur in geringer Ausdehnung vom Dotter abgehoben und hat nur an dieser Stelle den Charakter eines Cylinderepithels; die Zone des kompakten Entoderms ist dafür hier ausgedehnter und daran schließen sich die lose liegenden Zellen, die hier auch mehr Raum einnehmen als in der Mitte. Am Rande der Keimscheibe sehen wir die Ursprungsstätte des peripheren Mesoderms ${ }^{1}$ ) vollständig auf der ventralen Seite. Fig. 75, welche einen Schnitt anßerhalb der Embryonalanlage darstellt, zeigt bezuiglich des rom Dotter abgehobenen Theiles des Entoderms ähnliche Verhältmisse wie Fig. 74, hingegen geht derselbe hier ohne Übergang in das den Dotter bedeckende Plattenepithel iiber.

Spätere Stadien lehren, dass der gröBte Theil des in Fig. 73 noch nicht zu Cylinderepithel umgestalteten Entoderms noch dazu d. h. also zu gastralem Entoderm wird; das seitlich gelegene (wie in Fig. 74) wird es erst später in dem Maße, als der ventrale Darmverschluss fortschreitet; ein Theil der in losem Verbande stehenden Entodermzellen kann aber möglicherweise noch zu Dotterepithel werden. Damit hat sich dann die Scheidung des gastralen Entoderms rom Dotterentoderm vollzogen; beide Theile sind sowohl nach ihrer Form als nach ihrer Bestimmung von einander verschieden: ersteres ist Cylinderepithel, aus dem die Chorda, das Meso$\operatorname{derm}^{2}$ ) und der Darm entstehen, letzteres ist ein Plattenepithel, das den Dottersack auskleidet; identisch ist für beide Theile der Ursprung, wie ich im Gegensatz zu jenen Forschern betonen muss, die das gastrale Entoderm aus anderer Quelle herleiten wie das

1) Ich ziehe diesen von RüCKERT angewendeten Ausdruck dem RABL'schen speristomal vor, da letzterer von einer theoretischen Voraussetzung ausgeht, der ich nicht beipflichte.

2) Ich kann hier auf die Frage nach dem Ursprung des Mesoderms nicht näher eingehen, da mich dies von meinem Thema zu weit abführen wïrde. So weit ich aus den vorhandenen Mitosen schließen kann, wird das ganze Mesoderm vom Entoderm gebildet, und das.Ektoderm hat, trotzdem es seiner Lage nach ganz gut möglich wäre, an seiner Bildung keinen Antheil. Eine Grenze, wie sie LwoFF in seiner Fig. 43 zwischen Entoderm und Mesoderm zeichnet, habe ich niemals gefunden. 
Dotterentoderm. Die Verschiedenheit der Form, dass es in einem Falle ein Cylinderepithel, im anderen ein Plattenepithel ist, hat natürlich keine Bedeutung und hängt mit der verschiedenen Funktion zusammen. Ahnlich wird ja das Ektoderm, das ursprünglich in seiner ganzen Ausdehnung Cylinderepithel war, zuerst in seinem außerembryonalen Antheil und später, nachdem sich das Medullarrohr abgeschnürt hat, auch im Bereich des Embryos zu Plattenepithel.

Die weiteren Schicksale des: Entoderms sind bekannt; Chorda nnd Mesoderm werden ausgeschaltet; indem sich der Embryo über die Keimscheibe emporhebt, entsteht das eigentliche Darmlumen, und vom Kopfende her fortschreitend entsteht durch das Vorwachsen der seitlichen Entodermpartien die ventrale Darmwand. Gegenüber LwoFF möchte ich hervorheben, dass ich niemals das geringste Anzeichen dafür gefunden habe, dass in diesem Stadium von den Dotterkernen Entodermzellen gebildet werden; zwar liegen dort, wo das Entoderm sich an den Dotter ansetzt, meist mebrere kleine oder 'ein sehr umfangreicher Dotterkern; um dieselben findet sich häufig ein mit Gerinnsel erfüllter Hohlraum; einen Übergang der Kerne in Entoderm wird man aber niemals finden, und die Angaben LwoF's beruhen entweder auf schlechten Präparaten oder auf schlechten Beobachtungen.

Die Resultate der im Vorstehenden wiedergegebenen Beobachtungen sind kurz zusammengefasst folgende:

Das Ektoderm wird gebildet, indem der obere Theil desdurch die Furchngentstandenen soliden Haufens ron Furchungszellen sich zu einem Epithel zusammenfügt; die übrig bleibenden. Furchungstellen stellen das Entoderm, vor. Auch diese fïgen sich, wenn auch langsamer als das Ektoderm, zu einem Epithel zusammen, welches im Bereich der Embryonalanlage cylindrisch (gastrales Entoderm) a Berhalb desselben plattenförmig ist (Dotterentoderm). Irgend eine Einstülpung oder Einfaltung ist an der Bildung der Keimblätter nicht betheiligt.

Durch diese Processe kommt eine aus zwei Epithellagen gebildete Platte zu Stande, ein Stadium, das allerdings desshalb nicht rein zum Ausdruck kommt, weil die Bildung des Mesoderms schon begonnen hat, bevor die Epithelbildung im Entoderm abgeschlossen ist. Eine Schätzung, in welchem Verhältnis die Furchungszellen auf die beiden Keimblätter vertheilt werden, ist natürlich sehr 
schwierig; ich habe im Allgemeinen den Eindruck, als ob annähernd gleichviel Zellen auf jedes Keimblatt kämen.

\section{Schlussfolgerungen.}

Suchen wir nun die gewonnenen Thatsachen für die Frage zu verwerthen, ob bei Selachiern Gastrulation vorkommt oder nicht. Betrachten wir zunächst die Furchung; diese führt zur Bildung eines soliden Haufens von Furchungszellen, den man ganz gut als Morula bezeichnen kann. Bei Amphioxus kommt bekanntlich ein derartiges Stadium gar nicht vor, wie wahrscheinlich bei allen Eiern mit totaler und äqualer Furchung. Wir mïssen daher das Morulastadium der Selachier als ein cenogenetisches auffassen, das durch die Masse des Dotters bedingt ist. Der Process, durch den bei Selachiern sich die Zellen der Morula in zwei Schichten sondern, die den primären Keimblättern entsprechen, ist meines Erachtens nur mit der Scheidung der Keimblätter im achtzelligen Stadium bei Amphioxus zu vergleichen; da diese aber so außerordentlich viel später eintritt als bei Amphioxus, so ist sie dadurch natiirlich sehr modificirt. Man könnte zwar annehmen, dass bei Selachiern auch, ähnlich wie bei Amphioxus, die Scheidung der Keimblätter schon in einem frühen Stadium stattfinde, dass es aber eben kein Mittel gebe, dieselben von einander zu unterscheiden; diese Annahme glaube ich nach dem Verlauf der Furchung mit Bestimmtheit ausschließen zu können; denn erstens haben wir gesehen, dass ein Stadium, in dem alle Furchungszellen sich senkrecht zur Oberfläche theilen, bei Selachiern nicht existirt, und während der ganzen Furchung ist die Richtung der Theilungen ganz willkürlich, offenbar nur durch die Raumverhältnisse bedingt. Je weiter die Furchung vorschreitet, desto größer ist natürlich die Wahrscheinlichkeit, dass die Abkömmlinge der an der Oberfläche gelegenen Zellen zu Ektoderm, die der am Grunde liegenden zu Entoderm werden; die Zellen in der Mitte aber liefern sicher noch bis in die letzten Stadien Elemente für beide Keimblätter.

Die Frage, ob wir die Keimblätterbildung der Selachier als einen palingenetischen oder cenogenetischen Process ansehen wollen, wird wesentlich dadureh entschieden, wie man den damit verglichenen Process bei Amphioxus deutet; über Letzteres habe ich mich schon in der Einleitung geäußert. Selbst wenn man sich hier für die Annahme Bürscrnr's und daher für eine palingenetische 
Entstehung der Keimblätter entscheidet, so dürfte doch die Keimblattbildung der Selachier nur cum grano salis als palingenetische angesehen werden können, da wir hier von einem cenogenetisch bereits stark veränderten Stadium, der Morula, ausgehen müssen. Eine Einstuilpung oder Einfaltung lrommt bei der Keimblattbildung: nicht vor, wie ich schon wiederholt betont hatte; bei diesem Vorgange giebt es also nichts, was als Gastrulation gedeutet werden könnte.

Sehen wir, wie es in dieser Beziehung mit den späteren Stadien steht. Durch die Umwachsung des Dotters durch die Keimscheibe kommt allerdings schließlich eine Becherform des Keimes zu Stande, diese riihrt aber nicht ron einer aktiven Einkrümmung oder Finstiulpung der Keimscheibe her, sondern einfach davon, dass der Dotter aus physikalischen Gründen eine kugelige oder ellipsoidische Gestalt hat. In der Umwachsung des Dotters kann daher eine Gastrulation nicht erblickt werden.

Ebenso wenig wäre es möglich, dem ventralen Verschlusse des Darmrohres diese Deutung zu geben; denn wenn dieser Process auftritt, so handelt es sich gar nicht mehr um die Bildung eines Urdarms, sondern um die Bildung eines Theiles des definitiven Darms.

Nun könnte man sagen, dass die Gastrulation selbst zwar durch Cenogenese unterdrückt, das Gastrulastadium aber doch erhalten ist, wenn es auch nicht auf dem ursprünglichen Wege erreicht wird; prüfen wir, ob sich die Thatsachen mit dieser Auffassung in Übereinstimmung bringen lassen.

Ich habe hereits erwähnt, dass von den meisten Autoren der ganze Keimscheibenrand als Urmund betrachtet wird; dieser Rand hat aber nur die eine Eigenschaft des Urmunds, dass in ihm die beiden Keimblätter zusammenstoßen; in einen Urdarm kann er natürlich nicht führen, da er ja der Rand einer Scheibe ist; die Unzulässigkeit, die Grenze der Keimblätter als Urmund zu bezeichnen, habe ich schon in der Einleitung betont mit dem Hinweis darauf, dass z. B. bei Amphịoxus diese Grenze schon vor dem Gastrulastadium vorhanden ist.

Eine andere Beweisfuihrung benutzt $\mathrm{R}_{A B L}{ }^{1}$ ), um dem Keimscheibenrand der Selachier den Charakter als Urmund zuzuerkennen; er verweist auf einen Holzschnitt, der einen Sagittalschnitt durch einen 
Pristiurusembryo, entsprechend etwa meiner Fig. 65, wiedergiebt und bemerkt: „Es braucht wohl kaum erwähnt zu werden, dass der vordere Rand einer solchen Selachiergastrula (Fig. 24a) dem ventralen, der hintere $(A, 6)$ dem dorsalen Urmundrande der Amphioxusgastrula entspricht; wir brauchen uns ja nur den Nahrungsdotter weggenommen und die Gastrula zusammengebogen zu denken, um ein der Amphioxusgastrula ähnliches Bild zu bekommen. " Man bekommt ein ähnliches Bild, gewiss; aber weiter auch nichts und vor Allem kann aus dieser Ähnlichkeit nicht die Homologie des vorderen Keimscheibenrandes der Selachier mit dem ventralen Urmundrand des Amphioxus gefolgert werden; denn mit demselben Recht könnte man dann schließen, dass das Entoderm in der vorderen Hälfte der Selachierkeimscheibe homolog ist der ventralen Urdarmwand des Amphioxus, da es ja nach der „Umbiegung" dieselbe Lage einnimmt wie letzteres. Nun entsteht aber bei Selachiern daraus ein Theil des Dottersackentoderms; bei Amphioxus aber der größte Theil des definitiven Darms, also zwei gewiss nicht homologe Gebilde. Diese Art der Beweisfïhrung muss ich also ablehnen.

Andere Forscher, vor Allem O. HeRTwIG ${ }^{1}$, nehmen an, dass der Keimscheibenrand nur im Bereich der Embryonalanlage als Urmund anzusehen sei; hier findet sich ja in der That eine Öffnung, die in den Spalt rwischen dem gastralen Entoderm und dem Dotter führt; es fragt sich nun, ob wir diesen Spalt wirklich als Urdarmhöhle ansehen können. Das denselben an der dorsalen Seite begrenzende Entoderm zeigt allerdings große Übereinstimmung mit der dorsalen Urdarmwand des Amphioxus, in so fern auch hier aus derselben die Chorda und wenigstens ein Theil des Mesoderms (das axiale) entsteht. Anders steht es aber mit der ventralen Urdarmwand; diese liefert bei Amphioxus den größten Theil des künftigen Darms; bei Selachiern wäre dieselbe aber repräsentirt durch den Dotter; der in keiner Weise mit der ventralen Urdarmwand des Amphioxus homologisirt werden kann, da er weder die ventrale Darmwand liefert noch ïberhaupt einem Keimblatte zugerechnet werden kann. Die ventrale Urdarmwand würde also bei Selachiern einfach fehlen, und das, was bei Amphioxus aus der ventralen Urdarmwand gebildet wird, entsteht bei Selachiern erst in einem späteren Stadium durch einen Process, dem beim Amphioxus gar nichts entspricht. AuBerdem ist auch die Homologie 
zwischen dem mittleren Theil des gastralen Entoderms der Selachier und der dorsalen. Urdarmwand des Amphioxus keine vollständige, weil bei Selachiern nui das axiale. Mesoderm an dieser stelle entsteht, während die.Ursprungsstätte des peripheren Mesoderms bei Amphioxus überhaupt kein Homologon besitzt. Es hieße daher, wie ich glaube, den Theil für das Ganze nehmen, wenn man, auf diese theilweise Homologie gestiitzt, hier irgend etwas als Urdarm ansprechen wollte.

Nachdem ich alle diese Deutungen zurückweisen musste, bleibt mir nur die Annahme übrig, dass bei Selachiern die Gastrulation und das Gastrulastadi um fehlen; in Folge der großen Dottermenge ist dasselbe durch Cenogenese unterdrickt; wie wir uns vorstellen sollen, dass diese Unterdrückung im Laufe der Phylogenie allmählich stattgefunden hat, werde ich erst später zu beurtheilen suchen, wenn mir ein größeres Material zur Beurtheilung vorliegt.

Sehen wir aber nummehr von der Gastrulation ab und suchen wir einfach die Untersehiede, die zwischen Amphioxus und den Selachiern bezüglich der ersten Bildungsvorgänge herrschen, festzustellen. Bei Amphioxus ist die dritte Theilungsfurche äquatorial, bei Selachiern meridional, und auch in späteren Stadien tritt keine Theilung auf, die sich der dritten Theilung bei Amphioxus vergleichen ließe. Die Sonderung der primären Keimblätter, die bei Amphioxus im achtzelligen Stadium bereits vollzogen ist, tritt bei Selachiern viel später auf. Das Blastulastadium des Amphioxus fehlt, dafür findet sich eine Morula; eine Furchungshöhle findet sich nicht. Nach vollzogener Keimblattbildung finden wir eine aus zwei Zellschichten bestehende Platte. Die Einstïlpung des Entoderms, die bei Amphioxus zur Bildung einer von Entoderm ausgekleideten Höhle führt, fehlt bei Selachiern. Die ventrale Wand des Darms, die bei Amphioxus durch diese Einstülpung gegeben ist, muss bei Selachiern erst durch einen Process gebildet werden. der im Vergleich zu Amphioxus eine Neubildung ist. Dasselbe gilt vom peripheren Mesoderm, das an einer Stelle entspringt, die beim Amphioxus gar kein Homologon hat. In wie weit wir annehmen können, dass diese Unterschiede durch die Dottermenge bedingt sind, werdè ich in einer späteren Studie ausführen. 


\section{Erklärung der Abbildungen.}

Sämmtliche Figuren sind mit SEIBERT'schen Apochromaten and ABBÉ'schem Zeichenapparat in der Höhe des Objekttisches gezeichnet.

\section{Taf. XII.}

Fig. 1. 2. Seyllium eanicula. Keimscheibe im Befruchtungsstadium. Fig. 1. Richtungskörper. Fig. 2. Kern aus derselben. 1/750*

Fig. 3-5. Scyllium canicula. Etwas älteres Befruchtungsstadium als das vorige.

Fig. 3. Schnitt mit den in Vereinigung begriffenen beiden Vorkernen. 1/93. Fig. 4. Die beiden Vorkerne der vorigen Figur. 1/750. Fig. 5. Dotterkern. $1 / 750$.

Fig. 6. 7. Scyllium canicula. Zweizelliges Stadium. Fig. 6. Furehungskern. Fig. 7. Dotterkern. 1/750.

Fig. 8-16. Seyllium canicula. Vierzelliges Stadium. Fig. 8. Furchungskern. Fig. 9-16. Dotterkerne. $1 / 750^{*}$

Fig. 17-20. Scyllium canicula. Vierzelliges Stadinm. Fig. 17. Furchungskern. Fig. 18-20. Dotterkerne. 1/750-

Fig. 21-23. Scyllium canicula. Vierzelliges Stadium. Fig. 21. Furchungskern. Fig. 22. 23. Dotterkerne. $1 / 750$.

Fig. 24-28. Raja sp.? Vierzelliges Stadium. Fig. 24. Furchungskern 2. Fig. 25 bis 28. Dotterkerne. $1 / 750$.

Fig. 29. Scyllium catulus. Achtzelliges Stadium. Oberflächenansicht. $1 / 31$.

Fig. 30-38. Scyllium canicula. 16-zelliges Stadium. Fig. 30. Durchschnitt. 1/31 . Fig. 31-38. Dotterkerne.

Fig. 39-44. Scyllium canicula. 79 Furchungszellen. Fig. 39-41. Durchschnitte. 1/93. Fig. 42. 44. Dotterkerne. Fig. 43. Furchungskern.

\section{Taf. XIII.}

Fig. 45. 46. Seyllium canicula. Älteres Furchungsstadium. Durchschnitte.1/93. Fig. 47-57. Scyllium canicala. Älteres Furchungsstadium. Fig. 47. Durchschnitt. 1/93. Fig. 48-57. Dotterkerne. 1/750.

Fig. 58. Pristiurus. Dotterkern der Keimscheibe von Fig. 65-67. 1/750.

Fig. 59. Pristiurus. Dotterkern der Keimscheibe von Fig. 72-75. 1/750.

Fig. 60. Scyllium canicula. Embryo von $20 \mathrm{~mm}$ Länge. Dotterkerne aus dem Dottersack. 1/93. Fig. 60a. Dotterkern* der Fig. $601 / 750$.

Fig. 61. Scyllium canicula. Keimscheibe im Beginne der Keimblätterbildung. Sagittalschnitt. $1 / 93$.

Fig. 62. Pristiurus, etwas älteres Stadinm. Sagittalschnitt. 1/83.

Fig. 63. Pristiurus, etwas älteres Stadinm. Sagittalschnitt. 1/93.

\section{Taf. XIV.}

Fig. 64. Pristiurus, etwas älteres Stadium. Sagittalschnitt. 1/93.

Fig. 65-67. Pristiurns. Ektoderm bereits vollständig ausgebildet. Fig. 65. Hinterer 'Theil eines Sagittalschnittes in der Medianebene. Fig. 66. Seitlich geführter Sagittalschnitt. Fig. 67. Am Rande geführter Sagittalschnitt. $1 / 93$.

Fig. 68-71. Pristiurus, etwas älteres Stadium. Fig. 68. Oberflächenansicht des Embryonaltheils der Keimscheibe. 1/31. Fig. 69-71. Querschnitte. 1/93.

Fig. 72-75. Pristiurus. BALFouR's Stadium B. Fig. 72 . Medianer Sagittalschnitt. 1/31. Fig. 73. Hinterer Theil von Fig. 72. 1/93. Fig. 74. 75. Laterale Sagittalschnitte. $1 / 93$. 